\title{
BIOCHEMICAL VARIABILITY OF A DEEP LANDSLIDE-SET LAKE (LAKE TORTUM, ERZURUM/TURKEY)
}

\author{
FAKIOGLU, O. ${ }^{1 *}$ - NuHOGLU, A. ${ }^{2}-$ Egercioglu, M. N. ${ }^{1}-$ ACAR, C. ${ }^{1}$ \\ ${ }^{I}$ Department of Basic Science, Faculty of Fisheries, Atatürk University, Erzurum, Turkey \\ ${ }^{2}$ Department of Environmental Engineering, Faculty of Engineering, Atatürk University, \\ Erzurum, Turkey \\ ${ }^{*}$ Corresponding author \\ e-mail: ozden.fakioglu@atauni.edu.tr; phone: +90-442-231-2806; fax: +90-442-231-5881 \\ (Received 19 ${ }^{\text {th }}$ Apr 2020; accepted $29^{\text {th }}$ Jul 2020)
}

\begin{abstract}
Lake Tortum is a natural deep and landslide-set lake located in the northeast of Erzurum in the Eastern Anatolia Region of Turkey. The lake is under the influence of anthropogenic activity and pollution from agriculture. This paper, it was aimed to estimate the trophic level of Lake Tortum together with biological and some physicochemical parameters, as well as the external phosphorus load of this lake. The Lake was determined to be mesotrophic according to Secchi depth, total phosphorus concentration and chlorophyll-a tests. External phosphorus load was calculated above the critical phosphorus value determined for lakes. The lake has been shown in the eutrophication phase due to the nutrient inflow results exceeding the lake's loading capacity, the presence of some eutrophic species in the phytoplankton composition, and the periodic increase in blue-green algae. A total of 51 phytoplankton species were identified in the study period, belonging to 12 functional groups. The seasonal succession of dominant functional group is code LM (Ceratium hirundinella). The mean value of Q index in Tortum Lake was estimated as 1.88 which pointed out the tolerable ecological quality status.
\end{abstract}

Keywords: external phosphorus load, phytoplankton composition, $Q$ index, eutrophication

\section{Introduction}

Global warming and discrepancies in seasonal transitions diminish the freshwater resources each day. In addition to that, available freshwater resources face pollution threats with industrialization and increases in urban population. Determination of the nutrient status of a lake and uncovering the factors affecting it are very important in protecting the present water resources.

Lentic systems are impacted by the rock structure and climate of the catchment, or drainage basin, in which they are located, and each lake has its own dynamics (Wetzel, 2001). Lentic systems have a tendency to transform into ponds and swamps. That process takes a few hundred years in large lakes, but it can happen faster in their smaller counterparts (Tanyolac, 2009). Humanity has an impact on the evolution process of lakes as well. The main pollution sources for lake systems are urban, industrial, and agricultural activities (Metcalf and Eddy, 2002). In addition to those, nuclear power plants and poorly planned hydroelectric power plants are pollution sources. Organic and inorganic pollutants not only disrupt the physicochemical composition of lakes, but they also alter the biological structure (Coban, 2007). Pollutants and climate change have led to increases in eutrophication, fish deaths in some period (expecialy summer months), increasing of invasive species in lakes, carbon dioxide emission from lakes, and methane emission from sediments, as well as an increase in aquatic plants. Besides, excessive water diverted from lakes drops the water level of those lakes (Jessepsen et al., 2009). 
In addition to physicochemical parameters such as $\mathrm{N}, \mathrm{P}, \mathrm{Si}, \mathrm{Ca}$, and $\mathrm{Mg}$ content, phytoplankton communities and other aquatic organisms, such as fishes and macrophytes, are used as bioindicators in the determination of the trophic levels of lakes. Various studies have been conducted to determine the trophic levels of lakes by using phytoplankton communities and benthic algae (Thunmark, 1945; Nygaard, 1949; Lepistö and Rosenström, 1998). Those methods were not accepted by other researchers, however, because the phytoplankton communities exist as different species in different regions, and it was not understood whether pollution has any observable effect at all on the species distribution (Padisák et al., 2006). On the other hand, recent studies have illustrated that phytoplankton, macrophytes, benthic organisms, and fishes can be considered as biological parameters in the process of lake trophic level determination.

Reducing the nutrient level in lakes can improve water quality by controlling eutrophication. In addition to the restriction of external nutrients, the nutrients accumulated in the sediments - and especially phosphorus - should be removed in the process of nutrient reduction in lakes, because high nutrient accumulation can alter the biological structure. The most significant step in remediating a lake successfully and permanently is the determination of the corresponding nutrient load of the lake beforehand (Van Damme et al., 2007).

The aims of the study were to determine the trophic level of Lake Tortum, located in the Eastern Anatolian region of Turkey, and the effect of agricultural and other anthropogenic pollutants on the water quality and phytoplankton composition of the lake.

\section{Materials and Methods}

\section{Research area}

Lake Tortum, a natural landslide-barricaded lake, is situated in northeastern Erzurum. It is between $40^{\circ} 35^{\prime}$ and $40^{\circ} 39^{\prime}$ north latitude and at $41^{\circ} 38^{\prime}$ east longitude, at an altitude of $1012 \mathrm{~m}$ and $95 \mathrm{~km}$ from the city of Erzurum. It is about $8 \mathrm{~km}$ in length and $0.7 \mathrm{~km}$ in width, and the average depth is $100 \mathrm{~m}$. The lake has been utilized for touristic and fishing activities. The main water flow to the lake comes from Tortum Stream. The stream, which is approximately $50 \mathrm{~km}$ in length, starts in the Mescit, Y1ldızdağ, and Ereğli Mountains and collects all the water from a $1900 \mathrm{~km}^{2}$ catchment in the Tortum District and enters the lake. After its exit, it creates a large waterfall at the shoreline. Later, it merges with the Çoruh River (Kıvrak, 2006; Kıvrak and Gurbuz, 2010).

Research on identification of trophic level on the lake are limited. The first study was carried out in the lake in 1982 for determination of phytoplankton species and some physico-cemical water quality parameters. Another study has been performed on the lake between 2005-2006. In this study, it was investigated if there was changed in plankton species, and finally, in the 2012-2013 period, the structure of the lake's phytoplankton communities and the changes in some water quality parameters were investigated (Altuner, 1982; Kivrak, 2006; Fakıglu et al., 2014, 2018). With its $6.45 \mathrm{~km}^{2}$ lake area, Lake Tortum is a significant lake in Tortum Stream catchment $\left(1653 \mathrm{~km}^{2}\right)$. The average depth of the lake is $100 \mathrm{~m}$, and its volume is assumed to be $57.6 \mathrm{hm}^{3}$. The hydrological and morphometric measurements related to the lake are provided in Table 1. Some of the values in the table were provided from DSI (The General Directorate of State Hydraulic Works, Tur, Ministry of Agriculture and Forestry). 
Table 1. Seasonal variation of some water quality parameters andSecchi dept in Lake Tortum

\begin{tabular}{|c|c|c|c|c|c|c|c|c|c|c|c|c|}
\hline \multirow[t]{2}{*}{ Month } & \multicolumn{2}{|c|}{$\begin{array}{l}\text { Total Hardness } \\
\left(\mathrm{mgL}^{-1} \mathrm{CaCO}_{3}\right)\end{array}$} & \multicolumn{2}{|c|}{$\begin{array}{l}\text { Conductivity } \\
\left(\mathrm{mS} \mathrm{cm}^{-1}\right)\end{array}$} & \multicolumn{2}{|c|}{$\begin{array}{c}\text { Dissolved } \\
\text { oxygen }\left(\mathrm{mgL}^{-1}\right)\end{array}$} & \multicolumn{2}{|c|}{ pH } & \multicolumn{2}{|c|}{\begin{tabular}{|c|} 
Water \\
temperature $\left({ }^{\circ} \mathrm{C}\right)$
\end{tabular}} & \multicolumn{2}{|c|}{$\begin{array}{c}\text { Secchi dept } \\
(\mathrm{m})\end{array}$} \\
\hline & Mean & SD $^{*}$ & Mean & SD & Mean & SD & Mean & SD & Mean & SD & Mean & SD \\
\hline Feb & $137.56^{\mathrm{F}^{* *}}$ & 8.02 & $.261^{\mathrm{F}}$ & .002 & $7.91^{\mathrm{B}}$ & .39 & $8.35^{\mathrm{D}}$ & .06 & $4.34^{\mathrm{G}}$ & .16 & $3.13^{\mathrm{D}}$ & .06 \\
\hline Mar & $171.00^{\mathrm{C}}$ & 13.16 & $.279^{\mathrm{D}}$ & .003 & $6.57^{\mathrm{E}}$ & .35 & $8.40^{\mathrm{D}}$ & .06 & $6.09^{\mathrm{F}}$ & .59 & $4.30^{\mathrm{C}}$ & .61 \\
\hline Apr & $202.11^{\mathrm{A}}$ & 40.77 & $.285^{\mathrm{C}}$ & .007 & $8.38^{\mathrm{A}}$ & .66 & $8.52^{\mathrm{B}}$ & .08 & $7.13^{\mathrm{D}}$ & 1.06 & $2.15^{\mathrm{E}}$ & .74 \\
\hline May & $151.72^{\mathrm{E}}$ & 15.68 & $.281^{\mathrm{C}}$ & .015 & $7.20^{\mathrm{C}}$ & .39 & 8.35 & .05 & $10.33^{\mathrm{C}}$ & 2.87 & $4.07^{\mathrm{C}}$ & 2.01 \\
\hline Jun & $150.33^{\mathrm{E}}$ & 41.06 & $.262^{\mathrm{F}}$ & .024 & $7.00^{\mathrm{C}}$ & .58 & $8.53^{\mathrm{B}}$ & .10 & $10.63^{C}$ & 2.80 & $7.80^{\mathrm{A}}$ & 1.15 \\
\hline Jul & $189.22^{\mathrm{B}}$ & 16.77 & $.305^{\mathrm{B}}$ & .027 & $7.10^{\mathrm{C}}$ & 1.19 & $8.58^{\mathrm{A}}$ & .13 & $14.68^{\mathrm{A}}$ & 5.88 & $3.23^{\mathrm{D}}$ & 0.21 \\
\hline Aug & $161.44^{\mathrm{D}}$ & 20.76 & $.319^{\mathrm{A}}$ & .028 & $6.70^{\mathrm{D}}$ & 1.37 & $8.46^{\mathrm{C}}$ & .11 & $15.24^{\mathrm{A}}$ & 7.34 & $3.37^{\mathrm{D}}$ & 1.18 \\
\hline Sep & $177.50^{\mathrm{B}}$ & 29.17 & $.320^{\mathrm{A}}$ & .025 & $6.15^{\mathrm{D}}$ & .64 & $8.54^{\mathrm{B}}$ & .10 & $14.26^{\mathrm{A}}$ & 5.73 & $7.30^{\mathrm{A}}$ & .82 \\
\hline Oct & $179.17^{\mathrm{B}}$ & 27.17 & $.305^{\mathrm{B}}$ & .017 & $5.95^{\mathrm{F}}$ & .31 & $8.52^{\mathrm{B}}$ & .09 & $11.40^{\mathrm{B}}$ & 3.82 & $4.93^{\mathrm{B}}$ & .21 \\
\hline Nov & $157.94^{\mathrm{E}}$ & 9.47 & $.299^{\mathrm{B}}$ & .012 & $5.95^{\mathrm{F}}$ & .57 & $8.52^{\mathrm{B}}$ & .12 & $10.11^{\mathrm{C}}$ & 2.63 & $5.23^{\mathrm{B}}$ & .25 \\
\hline Dec & $159.89^{\mathrm{E}}$ & 13.60 & $.277^{\mathrm{D}}$ & .001 & $6.30^{\mathrm{E}}$ & .72 & $8.54^{\mathrm{B}}$ & .18 & $6.71^{\mathrm{E}}$ & .36 & $5.20^{\mathrm{B}}$ & 1.01 \\
\hline Jan & $165.50^{\mathrm{D}}$ & 12.70 & $.272^{\mathrm{E}}$ & .001 & $6.50^{\mathrm{E}}$ & .47 & $8.48^{\mathrm{C}}$ & .05 & $5.81^{F}$ & .04 & $3.73^{\mathrm{D}}$ & .42 \\
\hline
\end{tabular}

* Standard Deviation, ** The different capital letters in the same column show the differences between months $(\mathrm{p}<0.05)$

\section{Fieldwork and sampling}

Between February 2017 and January 2018, monthly water and plankton samples were taken in Lake Tortum (Erzurum). A total of 5 stations have been identified through the lake (3 stations) and Tortum Stream (2 stations) (Fig. 1). Three stations in the lake and in 5 different depths at each station (surface, $5 \mathrm{~m}, 10 \mathrm{~m}, 30 \mathrm{~m}$, and $40 \mathrm{~m}$ depth) samples were collected by Ruttner type sampler. Simultaneously, one station at the entrance of Tortum Stream to the lake and one station at its exit were taken water samples for investigation of extarnal phosphorus loading. Phytoplankton samples were taken with the plankton nets for identification of phytoplankton in the lake. To determine the number of phytoplankton, water samples with Lugol solution added, which were taken from both stations and depths of the lake, were brought to the laboratory with polyethylene containers. In order to determine the atmospheric phosphorus loading, presipitations coming to the location of the lake were collected in a sterile container every month and then transferred to the polyester sample bottles of $2 \mathrm{~L}$.

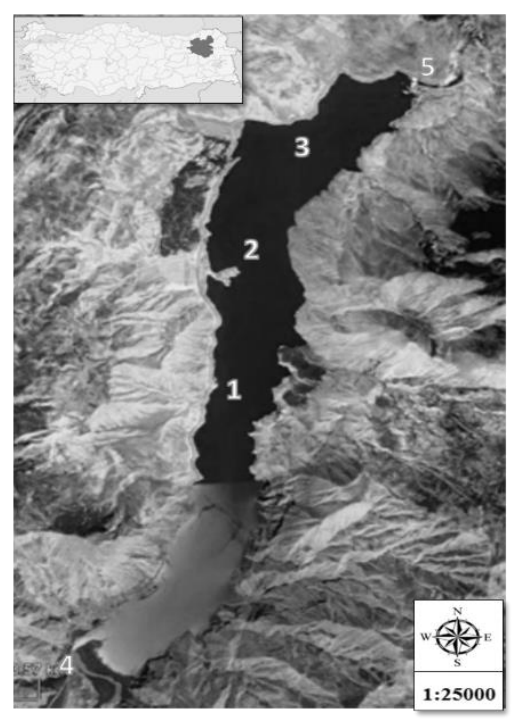

Figure 1. Location of Lake Tortum and stations 


\section{Identification and calculation of phytoplankton}

Phytoplankton species were identified under a binocular microscope with 100x and 400x magnification using a literature (Hustedt, 1930; Huber-Pestalozzi, 1938, 1942, 1950; Starmach, 1966; Prescott, 1973; Lind and Brook, 1980; Popovski and Pfiester, 1990; Cox, 1996; Komarek and Anagnostidis, 1999; John et al., 2002). The detected species have been checked on www.algaebase.com. Diatoms were investigated after precipitating the water samples with Lugol's solution addition; then nitric and sulfuric acids in equal volumes were utilized for boiling and acid-washing to remove the organics and expose the diatoms' silica skeletons (Round, 1953). For the enumeration of phytoplankton, water samples were first put into $10 \mathrm{~mL}$ graduated cylinders, and Lugol's solution was added and left overnight. After that, $3 \mathrm{~mL}$ of that solution was conveyed to phytoplankton enumeration rings, and the enumeration was done via an inverted microscope (Utermohl, 1958; Anonymous, 2003).

The Q phytoplankton assemblage index was estimated following Padisák et al. (2006), and ranged from 0 to 5 on a scale according to the WFD (World Framework Drictive) requirements. According to WFD's five grade evaluation system can be evaluated at $0-1$ : bad; between 1 and 2 as tolerable; 2-3: medium; between 3 and 4 as good, and 4-5: excellent quality (Padisák et al., 2006). For Lake Tortum, the factor numbers described for the Hungarian lake type 1 were used (Padisák et al., 2006). Phytoplankton species constituted more than $5 \%$ of total biomass were classified into functional groups according to Reynolds et al. (2002) and Padisák et al. (2009). The Water Framework Directive was adopted by the European Union in 2005. Turkey is a country in the process of integration into the European Union. Therefore, the rules have brought this directive has been implemented in Turkey. In this context, watershed-based study has been conducted in Turkey. In these studies, it was stated that the values developed for Hungarian lake type can be adapted in the use of Q index for in the evaluation of phytoplankton communities in Turkey, as well (Selek and Karaaslan, 2019).

\section{Water quality analyses and chlorophyll-a}

Water temperature, dissolved oxygen, $\mathrm{pH}$, conductivity (with YSI Multiparameter), and Secchi depth (with Secchi Disk, 20Ø) were measured in situ. Total hardness analyse in water samples were conducted according to Anonymous (1995). In TP (total phosphorus) analyses, the first part (digestion) was done by using the persulfate decomposition technique. Consequently, free orthophosphate phosphorus $\left(\mathrm{PO}_{4}-\mathrm{P}\right)$ was analyzed by the ascorbic acid method. Ammonia-nitrogen $\left(\mathrm{NH}_{3}-\mathrm{N}\right)$ was detected by the Nesslerization method. Nitrite-nitrogen $\left(\mathrm{NO}_{2}-\mathrm{N}\right)$ was detected by making use of the color formed with diazotization of sulfanilic acid (by nitrite) and the addition of the product to $\mathrm{N}$-1-naphthylethylenediamine dihydrochloride. Its absorbance was then measured via $523 \mathrm{~nm}$ wavelength light. Nitrate-nitrogen $\left(\mathrm{NO}_{3}-\mathrm{N}\right)$ concentration in water samples was determined by the yellow color formed after the nitrate's reaction with brucine sulfate; then the solution's absorbance was measured via $410 \mathrm{~nm}$ light (Anonymous, 1995). For chlorophyll $a$ detection, 1-L water samples were collected, and the combined water filtration system filtered them through a Whatman GF/C filter. The filtrate was left for 3 to 4 hours and decomposed. After that, it was left in $10 \mathrm{~mL}$ of $\% 90$ acetone solution, and the centrifuged extract's optical density was monitored in a spectrophotometer in wavelength of 630, 645, and $665 \mathrm{~nm}$ light (Strickland and Parsons, 1972). 


\section{External phosphorus loading}

The TP loading reaching the lake from the land (TPL, land loading) is equal to the multiplication of the entering waters' phosphorus export coefficients and the catchment area. The phosphorus export coefficient (Ep) is calculated from the TP carried by the streams in a year-flow rate $\mathrm{x}$ TP concentration $\left(\mathrm{kg} \cdot \mathrm{yr}^{-1}\right)$ divided by the area of the stream in the catchment calculated through planimeter from a standard topographic map (1:50.000) (Kirchner and Dillon, 1975).

$$
L L=\sum A s x E p
$$

LL: Land loading $\left(\mathrm{kg} \cdot \mathrm{yr}^{-1}\right)$,

As: Stream catchment area $\left(\mathrm{km}^{2}\right)$,

Ep: Phosphorus export coefficient $\left(\mathrm{kg} \cdot \mathrm{km}^{-2} \cdot \mathrm{yr}^{-1}\right)$.

The atmospheric phosphorus loding (AL) was calculated according to Dillon and Rigler (1975).

$$
A L=P \times T P p \times A o
$$

AL: Atmospheric loading ( $\left.\mathrm{kg} \mathrm{yr}^{-1}\right)$,

$\mathrm{P}$ : Annual precipitation $\left(\mathrm{mm} . \mathrm{yr}^{-1}\right)$,

TPp: Average TP concentration in rainwater $\left(\mathrm{mgL}^{-1}\right)$,

Ao: Surface area of the lake $\left(\mathrm{km}^{2}\right)$.

$$
N L=L L+A l
$$

NL: Natural loading $\left(\mathrm{kg} \cdot \mathrm{yr}^{-1}\right)$,

LL: Land loading $\left(\mathrm{kg} \cdot \mathrm{yr}^{-1}\right)$,

AL: Atmospheric loading ( $\left.\mathrm{kg} \cdot \mathrm{yr}^{-1}\right)$.

In Turkey, the phosphorus load coming from domestic wastewater has been determined to be 3-4 g/d/capita, according to the İller Bankası General Specification of Wastewater Treatment Plant Process (Ozden, 2002). As a result, artificial or domestic load was calculated as (DL).

$$
D L=N \times \frac{\frac{3.5 g}{\text { day }}}{\text { capita }} \times 365 \text { days }
$$

DL: Domestic load (kg. $\left.\mathrm{yr}^{-1}\right)$,

$\mathrm{N}$ : Basin population (individual).

The phosphorus load reaching the lake $\left(\mathrm{kg}_{\mathrm{gr}} \mathrm{yr}^{-1}\right)$ is equal to the sum of natural and domestic loads.

$$
T P L=N L+D L
$$

TPL: Total phosphorus load (kg. $\left.\mathrm{yr}^{-1}\right)$,

NL: Natural load $\left(\mathrm{kg} \cdot \mathrm{yr}^{-1}\right)$,

DL: Domestic load $\left(\mathrm{kg} \cdot \mathrm{yr}^{-1}\right)$.

On the other hand, the total phosphorus loading factor is equal to the TP load divided by the lake's surface area. 


$$
L p=T P L \div A o
$$

Lp: Total phosphorus loading factor $\left(\mathrm{kg} \cdot \mathrm{km}^{-2} \cdot \mathrm{yr}^{-1}\right)$,

TPL: Total phosphorus load (kg. $\left.\mathrm{yr}^{-1}\right)$,

Ao: Surface area of the lake $\left(\mathrm{km}^{2}\right)$.

Vollenweider (1976), emphasized that water residence time is also important in the critical load formula. It was found that the critical phosphorus concentration should be between $10 \mathrm{mg} / \mathrm{m}^{3}$ and $20 \mathrm{mg} / \mathrm{m}^{3}$. According to that, the following formula is used to calculate critical phosphorus loading:

$$
\text { Lcritical }=10 q(1+\sqrt{t w})
$$

Lcritical: Critical phosphorus loading $\left(\mathrm{mg} \cdot \mathrm{m}^{-2} \cdot \mathrm{yr}^{-1}\right)$,

q: Flushing rate $\left(\mathrm{m}_{\mathrm{yr}} \mathrm{yr}^{-1}\right)$,

tw: Hydraulic retention time (year).

The permit water loading $\left(\mathrm{g} \cdot \mathrm{m}^{-2} \cdot \mathrm{yr}^{-1}\right)$ and the critical loading value $\left(\mathrm{g} \cdot \mathrm{m}^{-2} \cdot \mathrm{yr}^{-1}\right)$ was determined by the equations, used to be flushing rate (q, m. $\mathrm{yr}^{-1}$ ), given below (Chapra and Tarapchak, 1976):

$$
\begin{aligned}
& \text { Lpermit }=0.011(q+12.4) \\
& \text { Lcritical }=0.025(q+12.4)
\end{aligned}
$$

Critical phosphorus loading could also be calculated by using field phosphorus loading $\left(\mathrm{g} \cdot \mathrm{m}^{-2} \cdot \mathrm{yr}^{-1}\right)$, chlorophyll-a $\left(\mathrm{mgL}^{-1}\right)$ and flushing rate $\left(\mathrm{q}, \mathrm{m} \cdot \mathrm{yr}^{-1}\right)($ Chapra and Tarapchak, 1976):

$$
\text { Lcritical }=0.0055(\text { chl } a)^{0.69}(q+12.4)
$$

\section{Statistics}

The monthly, stationary, and depth-related changes in the data taken from the three selected stations' surface, $5 \mathrm{~m}, 10 \mathrm{~m}, 20 \mathrm{~m}, 30 \mathrm{~m}$, and $40 \mathrm{~m}$ depths were examined with IBM SPSS 20 software. To understand the collective effect of the stations and seasons, water temperature, dissolved oxygen, $\mathrm{pH}$, conductivity, Secchi disk, total hardness, $\mathrm{NH}_{3}-$ $\mathrm{N}, \mathrm{NO}_{2}-\mathrm{N}, \mathrm{NO}_{3}-\mathrm{N}, \mathrm{TP}$ and $\mathrm{PO}_{4}-\mathrm{P}$ were analyzed by the use of three-factorial Analysis of Variance (ANOVA). The Duncan test was employed to determine the intergroup differences. Canonical Correspondence Analysis was done with XLSTAT software.

\section{Results}

\section{Physico-chemical parameters of Lake Tortum}

The average depth of the Lake Tortum (100 m) was provided from DSI (The General Directorate of State Hydraulic Works, Tur, Ministry of Agriculture and Forestry). The depth of Secchi was determined between $3 \mathrm{~m}$ and $5 \mathrm{~m}$ throughout the year. The lowest value $(2.15 \pm 0.74 \mathrm{~m})$ of Secchi depth was measured in April and the highest value $(7.80 \pm 1.51 \mathrm{~m})$ in June during the clean water phase. The mean Secchi depth was $4.42 \mathrm{~m}$. 
Stationary and depth-related changes in water temperature, dissolved oxygen, $\mathrm{pH}$, conductivity and total hardness values were statistically significant $(\mathrm{p}<0.05)$. Water temperature, dissolved oxygen, $\mathrm{pH}$, conductivity, and total hardness mean values in the lake were $10.64{ }^{\circ} \mathrm{C}, 6.81 \mathrm{mgL}^{-1}, 8.47,0.289 \mathrm{mS} \mathrm{cm}^{-1}$, and $166 \mathrm{mgL}^{-1} \mathrm{CaCO}_{3}$, respectively (Table 1).

The catchment area of Lake Tortum, shows microclimate characteristics (Duman, 2009), so the lake not ice-covered in winter, and the mean water temperature in the winter months was measured as $6{ }^{\circ} \mathrm{C}$. The thermal stratification in spring and autumn periods was weak, but a considerable stratification was observed in summer. Thus, Lake Tortum belongs to the warm monomictic lake class in Wetzel's (2001) classification of the lakes according to their stratification.

Detected lowest dissolved oxygen value was $\left(5.6 \mathrm{mgL}^{-1}\right)$ in September and the highest value $\left(8.65 \mathrm{mgL}^{-1}\right)$ in April. The dissolved oxygen value in the lake water supply and the drainage water of the lake was also higher than the lake and the average minimum and maximum values were found to be $5.06 \mathrm{mgL}^{-1}$ and $11.03 \mathrm{mgL}^{-1}$, respectively (Table 1). The lowest water temperature value was detected at the $1^{\text {st }}$ station $\left(4.1^{\circ} \mathrm{C}\right)$ in February, at a depth of 5 and $10 \mathrm{~m}$, and the highest water temperature value at the $1^{\text {st }}$ station $\left(25.4^{\circ} \mathrm{C}\right)$ on the surface in August. Depending on the depth of the Tortum Lake water temperature, a significant stratification was detected in the summer and autumn seasons. In spring, the water temperature value on the surface was measured at $10.26^{\circ} \mathrm{C}$, and as the depth increased, a decrease of $1{ }^{\circ} \mathrm{C}$ was observed in the water temperature value. During the winter period, the water temperature value ranged between $5.37^{\circ} \mathrm{C}$ and $5.8^{\circ} \mathrm{C}$, respectively, at depths of 0-40 $\mathrm{m}$. At the stations that feed the lake and discharge the lake water, the water temperature value was determined as the lowest $3.4{ }^{\circ} \mathrm{C}$ and $4.8{ }^{\circ} \mathrm{C}$, the highest $24.1^{\circ} \mathrm{C}$ and $25.1^{\circ} \mathrm{C}$, respectively.

The mean $\mathrm{pH}$ value was measured as $8.48 \pm 0.12$. According to Turkish Environmental Legislation Inland Water Resources Classification, the lake was identificated as first class waters quality (Anonymous, 2012). $\mathrm{pH}$ was measured in June at its lowest value in the $1^{\text {st }}$ station (8.0) and the highest value in February at the depth of $10 \mathrm{~m}(8.9)$ in June. At the $2^{\text {nd }}$ station, the lowest value (8.1) was found at the surface in July at the surface depth in July (8.79). The lowest value at the $3^{\text {rd }}$ station (8.32) was determined at the depth of May $20 \mathrm{~m}$, the highest value (8.7) at $0 \mathrm{~m}$ and at a depth of $5 \mathrm{~m}$ in July (Table 1).

The difference between TP, $\mathrm{PO}_{4}-\mathrm{P}, \mathrm{NH}_{3}-\mathrm{N}, \mathrm{NO}_{2}-\mathrm{N}$ and $\mathrm{NO}_{3}-\mathrm{N}$ concentration values between stations, months and depths was found to be statistically significant $(\mathrm{p}<0.05)$. $\mathrm{TP}, \mathrm{PO}_{4}-\mathrm{P}, \mathrm{NH}_{3}-\mathrm{N}, \mathrm{NO}_{2}-\mathrm{N}$, and $\mathrm{NO}_{3}-\mathrm{N}($ mean $\pm \mathrm{SD})$ were $0.31 \pm 0.03 \mathrm{mgL}^{-1}, 41.05 \pm$ $2.65 \mu \mathrm{gL}^{-1}, 0.14 \pm 0.02 \mathrm{mgL}^{-1}, 0.55 \pm 0.08 \mathrm{mgL}^{-1}$, and $1.10 \pm 0.02 \mathrm{mgL}^{-1}$, respectively.

$\mathrm{TP}$ values were $0.33 \pm 0.03 \mathrm{mgL}^{-1}, 0.34 \pm 0.04 \mathrm{mgL}^{-1}$, and $0.27 \pm 0.03 \mathrm{mgL}^{-1}$ in the first, second, and third stations, respectively. $\mathrm{PO}_{4}-\mathrm{P}$ showed its lowest value $\left(0.0 \pm 0.0 \mu \mathrm{gL}^{-1}\right)$ at all stations except for the months of July, August, and January. The highest values in the first, second, and third stations were $77.84 \pm 0.09 \mu \mathrm{gL}^{-1}$, $39.20 \pm 0.01 \mu \mathrm{gL}^{-1}$, and $47.16 \pm 0.02 \mu \mathrm{gL}^{-1}$, respectively (Table 2, Table 3).

For differences of $\mathrm{NH}_{3}-\mathrm{N}$ at the stations, the lowest value at the first station was found in January in all depths; on the other hand, the highest value was $0.25 \pm 0.01 \mathrm{mgL}^{-1}$ at $10 \mathrm{~m}, 30 \mathrm{~m}$, and $40 \mathrm{~m}$ depths in March. At the second station, the lowest value was $0.06 \pm 0.01 \mathrm{mgL}^{-1}$ on the surface in June, and the highest value was $0.43 \pm 0.02 \mathrm{mgL}^{-1}$ at $40 \mathrm{~m}$ depth in January. At the third station, the lowest value was $0.0 \pm 0.0 \mathrm{mgL}^{-1}$ at the surface in August, and the highest value $0.45 \pm 0.02 \mathrm{mgL}^{-1}$ was at $5 \mathrm{~m}$ depth in January (Table 4). 
Table 2. Change of Total Phosphorus (TP) values depending on months, stations and depth on Tortum Lake (Mean $\left.\pm S D, m g L^{-1}\right)(n=4)$

\begin{tabular}{|c|c|c|c|c|c|c|c|c|c|c|c|c|c|}
\hline St & epth & Feb & Mar & Apr & May & Jun & Jul & Aug & Sep & Oct & Nov & Dec & Jan \\
\hline \multirow{6}{*}{1} & 0 & $0.00 \pm 0.0^{\mathrm{Gdg} g^{* *}}$ & $04 \pm 0.02^{\mathrm{Fcd}}$ & $08 \pm 0.02^{\mathrm{Fa} a}$ & $0.0001 \pm 0.0^{\mathrm{Gaa} a}$ & $1.16 \pm 0.0^{\mathrm{Ad} c}$ & $96 \pm 0.02^{\mathrm{Aa} a}$ & $0.24 \pm 0.01^{\mathrm{Dac}}$ & $0.28 \pm 0.01^{\mathrm{Db} f}$ & $0.20 \pm 0.01^{\mathrm{Ea} a}$ & $0.80 \pm 0.05^{\mathrm{Ba} a}$ & $0.28 \pm 0.02^{\text {Dde }}$ & \\
\hline & 5 & $0.02 \pm 0.01^{\mathrm{Ff} f}$ & $.06 \pm 0.02^{\mathrm{Fc} d}$ & $24 \pm 0.02^{\mathrm{D} c b}$ & $0.0001 \pm 0.0^{\mathrm{Gaa} a}$ & $0.81 \pm 0.03^{\mathrm{Ab} b}$ & $0.70 \pm 0.02^{\mathrm{Bcc}}$ & $13 \pm 0.01^{\mathrm{Eb} d}$ & $0.44 \pm 0.01^{\text {Cae }}$ & $0.26 \pm 0.01^{\mathrm{Da} a}$ & $0.44 \pm 0.01^{\mathrm{Cb} b}$ & $0.47 \pm 0.02^{\mathrm{Ccd}}$ & $0.01^{\mathrm{Db} d}$ \\
\hline & 10 & $00 \pm 0.0^{\mathrm{Gd} g}$ & $0.20 \pm 0.02^{\mathrm{Db} c}$ & $08 \pm 0.01^{\mathrm{Ed} c}$ & $0.0001 \pm 0.0^{\mathrm{Fa} a}$ & $0.92 \pm 0.04^{\mathrm{Aa} a}$ & $0.80 \pm 0.01^{\mathrm{Bb} b}$ & $0.07 \pm 0.01^{\mathrm{Ece}}$ & $0.46 \pm 0.01^{\text {Еae }}$ & $0.0 \pm 0.0^{\mathrm{G} b b}$ & $0.40 \pm 0.01^{\mathrm{Ccd}}$ & $0.93 \pm 0.01^{\mathrm{Ad} d}$ & $0.24 \pm 0.01^{\mathrm{Dc} f}$ \\
\hline & 20 & $0.04 \pm 0.01^{\mathrm{Fb} e}$ & $.20 \pm 0.02^{\mathrm{Db} c}$ & $.36 \pm 0.02^{\mathrm{Cb} b}$ & $0.0001 \pm 0.0^{\mathrm{Ga} a}$ & $0.48 \pm 0.03^{\mathrm{Bed}}$ & $0.90 \pm 0.02^{\mathrm{Aa} a}$ & $0.04 \pm 0.01^{\mathrm{Fce}}$ & $0.49 \pm 0.01^{\mathrm{Dad}}$ & $0.16 \pm 0.01^{\mathrm{E} c d}$ & $0.27 \pm 0.01^{\mathrm{Ed} e}$ & $0.24 \pm 0.02^{\mathrm{Bb} x}$ & $0.17 \pm 0.01^{\mathrm{Db} d}$ \\
\hline & 30 & & $.40 \pm 0.02^{\mathrm{Ca} b}$ & $.47 \pm 0.02^{\mathrm{Ca} a}$ & $0.0001 \pm 0.0^{\mathrm{Ga} a} \mid$ & $.59 \pm 0.03^{\mathrm{Bc} d}$ & $1.03 \pm 0.05^{\mathrm{Aa} a}$ & $0.04 \pm 0.01^{\mathrm{Fce}}$ & $0.52 \pm 0.01^{\mathrm{Bad}}$ & $0.0 \pm 0.0^{\mathrm{Gcd}}$ & $0.16 \pm 0.01^{\text {Ede }}$ & $0.56 \pm 0.01^{\mathrm{Bb} c}$ & $0.25 \pm 0.02^{\mathrm{Db} d}$ \\
\hline & 40 & & $40 \pm 0.02^{\mathrm{Ca} b}$ & $32 \pm 0.02^{\mathrm{Gb} b}$ & $0.0001 \pm 0.0^{\mathrm{Ga} a}$ & $0.40 \pm($ & $0.85 \pm 0.05^{\mathrm{Ab} b}$ & $0.29 \pm 0.01^{\mathrm{Cb} b}$ & $0.43 \pm 0.01^{\mathrm{Ba} e}$ & $0.27 \pm 0.01^{\mathrm{Ca} a}$ & $0.27 \pm 0.01^{\mathrm{Ccd}}$ & & \\
\hline \multirow{6}{*}{2} & 0 & & $0.52 \pm 0.02^{\mathrm{Ca} a}$ & $0.0001 \pm 0.0^{\mathrm{Gad}}$ & $0.0001 \pm 0.0^{\mathrm{Ga} a}$ & $.05^{\mathrm{Aa} a}$ & $0.72 \pm 0.05^{\mathrm{Bb} c}$ & $0.90 \pm 0.01^{\mathrm{Aa} a}$ & $0.60 \pm 0.01^{\mathrm{Ab} c}$ & $0.11 \pm$ & $0.07 \pm 0.0$ & $0.44 \pm 0.02^{\mathrm{Db} d}$ & 0.12 \\
\hline & 5 & $0.00 \pm 0.0^{\mathrm{Gd} c}$ & $0.41 \pm 0.02^{\mathrm{Cb} b}$ & $0.0001 \pm 0.0^{\mathrm{Gad}}$ & $0.0001 \pm 0.0^{\mathrm{Ga} a}$ & $0.91 \pm 0.03^{\mathrm{Aa} a}$ & $0.92 \pm 0.04^{\mathrm{Aa} a}$ & $0.16 \pm 0.01^{\mathrm{Eb} d}$ & $0.50 \pm 0.01^{\mathrm{Bc} d}$ & $0.19 \pm 0.01^{\mathrm{Ea} b}$ & $0.2 \pm 0.01^{\mathrm{Fce}}$ & $0.5 \pm 0.01^{\mathrm{Fd} f}$ & $0.31 \pm 0.02^{\mathrm{Dac}}$ \\
\hline & 10 & $0.03 \pm 0.0^{\mathrm{Fb} e}$ & $0.16 \pm 0.02^{\mathrm{Ecc}}$ & $0.0001 \pm 0.0^{\mathrm{Gad}}$ & $0.0001 \pm 0.0^{\mathrm{Ga} a}$ & $0.52 \pm 0.03^{\mathrm{Ccd}}$ & $0.95 \pm 0.04^{\mathrm{Aa} a}$ & $0.18 \pm 0.01^{\mathrm{Eb} d}$ & $0.68 \pm 0.04^{\mathrm{Bac}}$ & $0.10 \pm 0.01^{\mathrm{Cb} b}$ & $0.52 \pm 0.01^{\mathrm{Ca} b}$ & $0.32 \pm 0.01^{\text {Dce }}$ & $0.36 \pm 0.03^{\mathrm{Dac}}$ \\
\hline & 20 & $0.00 \pm 0.00^{\mathrm{Fd} g}$ & $.12 \pm 0.02^{\mathrm{Ecc}}$ & $0.0001 \pm 0.0^{\mathrm{Fad}}$ & $0.0001 \pm 0.0^{\mathrm{Faa} a}$ & $0.40 \pm 0.03^{\text {Dde }}$ & $1.13 \pm 0.06^{\mathrm{Ba} a}$ & $0.00 \pm 0.0^{\mathrm{Fd} f}$ & $0.72 \pm 0.04^{\mathrm{Ca} b}$ & $0.15 \pm 0.01^{\mathrm{Ea} b}$ & $0.12 \pm 0.01^{\mathrm{Eb} e}$ & $2.08 \pm 0.05^{\mathrm{Aa} a}$ & $0.15 \pm 0.02^{\mathrm{Eb} e}$ \\
\hline & 30 & $0.03 \pm 0.01^{\mathrm{Fb} e}$ & $0.19 \pm 0.02^{\mathrm{E} c c}$ & $0.0001 \pm 0.0^{\mathrm{Gad}}$ & $0.0001 \pm 0.0^{\mathrm{Ga} a}$ & $0.55 \pm 0.03^{\mathrm{Bc} d}$ & $0.76 \pm 0.03^{\mathrm{Abc}}$ & $0.02 \pm 0.01^{\mathrm{Fce}}$ & $0.70 \pm 0.04^{\mathrm{Aa} b}$ & $0.0 \pm 0.0^{\mathrm{Gd} d}$ & $0.00 \pm 0.0^{\mathrm{Gd} g}$ & $0.35 \pm 0.02^{\mathrm{Cce}}$ & $0.29 \pm 0.02^{\mathrm{Dad}}$ \\
\hline & 40 & $00 \pm 0.0^{\mathrm{Gd} g}$ & $0.21 \pm 0.02^{\mathrm{Ccc}}$ & $0.0001 \pm 0.0^{\mathrm{Fad}}$ & $0.0001 \pm 0.0^{\mathrm{Fa} a}$ & $0.63 \pm 0.03^{\mathrm{Bb} c}$ & $0.76 \pm 0.03^{\mathrm{Ab} c}$ & $0.04 \pm 0.01^{\mathrm{Ece}}$ & $0.79 \pm 0.04^{\mathrm{Aa} b}$ & $0.06 \pm 0.01^{\mathrm{Ecc}}$ & $0.12 \pm 0.01^{\mathrm{Db} e}$ & $0.56 \pm 0.01^{\mathrm{Bb} c}$ & $0.19 \pm 0.01^{\mathrm{Cb} e}$ \\
\hline \multirow{6}{*}{3} & 0 & & $0.23 \pm 0.01^{\mathrm{Eac}}$ & $0.0001 \pm 0.0^{\mathrm{Gad}}$ & $0.0001 \pm 0.0^{\mathrm{Ga} a}$ & $0.6 \pm 0.03^{\mathrm{Fd} f}$ & $0.75 \pm 0.03^{\mathrm{Ab} c}$ & $0.23 \pm 0.01^{\mathrm{Db} c}$ & $0.57 \pm 0.02^{\mathrm{Bad}}$ & $0.15 \pm 0.01^{\mathrm{Eb} b}$ & $0.00 \pm 0.0^{\mathrm{Gc} g}$ & & $0.13 \pm 0.01^{\mathrm{Eb} e}$ \\
\hline & 5 & $0.05 \pm 0.01^{\mathrm{Fb} c}$ & $0.25 \pm 0.01^{\mathrm{Dac}}$ & $0.0001 \pm 0.0^{\mathrm{Gad}}$ & $0.0001 \pm 0.0^{\mathrm{Ga} a}$ & $0.69 \pm 0.03^{\mathrm{Bb} c}$ & $0.94 \pm 0.04^{\mathrm{Aa} a}$ & $0.37 \pm 0.01^{\mathrm{Ca} b}$ & $0.60 \pm 0.01^{\mathrm{Bac}}$ & $0.12 \pm 0.01^{\mathrm{Eb} b}$ & $0.12 \pm 0.01^{\text {Eae }}$ & $0.36 \pm 0.01^{\mathrm{Cb} e}$ & $0.11 \pm 0.01^{\mathrm{Eb} e}$ \\
\hline & 10 & $0.06 \pm 0.0^{\mathrm{Eb} b}$ & $0.06 \pm 0.01^{\mathrm{Ec} d}$ & $0.0001 \pm 0.0^{\mathrm{Fad}}$ & $0.0001 \pm 0.0^{\mathrm{Fa} a}$ & $0.78 \pm 0.03^{\mathrm{Ba} b}$ & $0.93 \pm 0.04^{\mathrm{Aa} a}$ & $0.31 \pm 0.01^{\mathrm{Ca} b}$ & $0.40 \pm 0.01^{\mathrm{Cb} e}$ & $0.0 \pm 0.01^{\mathrm{Fd} d}$ & $0.06 \pm 0.01^{\mathrm{Eb} e}$ & $0.36 \pm 0.02^{\mathrm{Cb} e}$ & $0.16 \pm 0.01^{\mathrm{Db} e}$ \\
\hline & 20 & $0.08 \pm 0.00^{\mathrm{Aa} a}$ & $0.15 \pm 0.02^{\mathrm{Eb} c}$ & $0.0001 \pm 0.0^{\mathrm{Ga} d}$ & $0.0001 \pm 0.0^{\mathrm{Gaa} a}$ & $0.47 \pm 0.02^{\mathrm{Cce}}$ & $1.01 \pm 0.05^{\mathrm{Aa} a}$ & $0.24 \pm 0.01^{\mathrm{Dbc}}$ & $0.59 \pm 0.01^{\mathrm{Bad}}$ & $0.04 \pm 0.01^{\mathrm{Fcc}}$ & $0.12 \pm 0.01^{\text {Eae }}$ & $0.34 \pm 0.03^{\mathrm{Db} e}$ & $0.04 \pm 0.0^{\mathrm{Fc} f}$ \\
\hline & 30 & $0.08 \pm 0.0^{\mathrm{Fa} a}$ & $0.06 \pm 0.01^{\mathrm{Fc} d}$ & $0.0001 \pm 0.0^{\mathrm{Gad}}$ & $0.0001 \pm 0.0^{\mathrm{Ga} a} \mid$ & $0.10 \pm 0.01^{\mathrm{Ed} f}$ & $0.96 \pm 0.04^{\mathrm{Aa} a}$ & $0.05 \pm 0.01^{\mathrm{Fce}}$ & $0.47 \pm 0.01^{\mathrm{Ca} e}$ & $0.24 \pm 0.01^{\mathrm{Da} a}$ & $0.04 \pm 0.01^{\mathrm{Fb} f}$ & $0.54 \pm 0.02^{\mathrm{Bac}}$ & $0.25 \pm 0.02^{\mathrm{Db} d}$ \\
\hline & 40 & $0.02 \pm 0.01^{\mathrm{Dd} e}$ & $0.16 \pm 0.05^{\mathrm{Cb} c}$ & $0.00 \pm 0.00^{\text {Ebe }}$ & $0.0001 \pm 0.0^{\text {Еa } a}$ & $0.49 \pm 0.03^{\text {Bce }}$ & $0.90 \pm 0.04^{\mathrm{Aa} a}$ & $0.23 \pm 0.01^{\mathrm{Cb} c}$ & $0.50 \pm 0.01^{\mathrm{Bad}}$ & $0.04 \pm 0.01^{\mathrm{Dcc}}$ & $0.12 \pm 0.01^{\mathrm{Ca} e}$ & $0.56 \pm 0.02^{\mathrm{Bac}}$ & $0.47 \pm 0.03^{\mathrm{Ca} b}$ \\
\hline
\end{tabular}

** A, B , C , D ..: Capital letters show the difference between the months for each station and the difference between the months carrying different capital letters on the same line is statistically significant $(\mathrm{p}<0.05)$

$\mathrm{a}, \mathrm{b}, \mathrm{c}, \mathrm{d} . .:$ Lower case letters show the difference between the depths for each station and the difference between the depths carrying different lower case letters in the same line is statistically significant $(\mathrm{p}<0.05)$,

$\mathrm{a}, \mathrm{b}, \mathrm{c}, \mathrm{d}$..: Italic letters show the difference between stations for each station and the difference between the stations carrying different italic letters in the same line is statistically significant $(\mathrm{p}<0.05)$ 
Table 3. Change of Orthophosphate Phosphorus $\left(\mathrm{PO}_{4}-\mathrm{P}\right)$ values depending on months, stations and depth on Tortum Lake $\left(\mathrm{Mean} \pm S D, m g L^{-1}\right)(n=4)$

\begin{tabular}{|c|c|c|c|c|c|c|c|c|c|c|c|c|c|}
\hline St & Depth & Feb & Mar & Apr & May & Jun & Jul & Aug & Sep & Oct & Nov & Dec & Jan \\
\hline & 0 & $.00 \pm 0.0^{\mathrm{Db} a}$ & $82 \pm 0.02^{\mathrm{Cac}}$ & $17.61 \pm 0.01^{\mathrm{Bd} c}$ & $13.64 \pm 0.08^{\mathrm{Bb}}$ & $18.18 \pm 0.01^{\mathrm{Bb} c}$ & $25.00 \pm 0.01^{\mathrm{Ab} b}$ & $0.00 \pm 0.0^{\mathrm{Bd} e}$ & $1.14 \pm 0.01^{\mathrm{Cb} b}$ & $0.00 \pm 0.0^{\mathrm{D} c c}$ & $6.25 \pm 0.02^{\mathrm{Cb} b}$ & $13.07 \pm 0.09^{\mathrm{Ba}}$ & $\begin{array}{ll}b & 9.09 \pm 0.01^{\mathrm{Cc} b}\end{array}$ \\
\hline & 5 & $1.14 \pm 0.01^{\mathrm{Ca} b}$ & $0.00 \pm 0.0^{\mathrm{Dd} d}$ & $9.09 \pm 0.02^{\mathrm{Ce} d}$ & $27.27 \pm 0.09^{\mathrm{Aac}}$ & $a 17.61 \pm 0.01^{\mathrm{Bb} c}$ & $30.11 \pm 0.02^{\mathrm{Aa} b}$ & $10.80 \pm 0.05^{\mathrm{Bb} c}$ & $0.00 \pm 0.0^{\mathrm{Dcc}}$ & $10.80 \pm 0.05^{\mathrm{Bac}}$ & $4.55 \pm 0.02^{\mathrm{Cb} b}$ & $13.07 \pm 0.08^{\mathrm{Ba}}$ & $4.55 \pm 0.01^{\mathrm{Cc} b}$ \\
\hline & 10 & $0.00 \pm 0.0^{\mathrm{Da} b}$ & $0.00 \pm 0.0^{\mathrm{Dd} d}$ & $0.57 \pm 0.01^{\mathrm{Dfe} e}$ & $0.00 \pm 0.0^{\mathrm{Dcd} d}$ & $25.00 \pm 0.01^{\mathrm{Aal}}$ & $\mathrm{b} 34.09 \pm 0.02^{\mathrm{Aa} a}$ & $6.82 \pm 0.05^{\mathrm{Cc} d}$ & $0.00 \pm 0.0^{\mathrm{Dcc}}$ & $0.00 \pm 0.0^{\mathrm{D} c c}$ & $9.09 \pm 0.02^{\mathrm{Cb} b}$ & $14.20 \pm 0.07^{\mathrm{Ba}}$ & $b \mid 10.80 \pm 0.01^{\mathrm{Bb} b}$ \\
\hline 1 & 20 & $0.00 \pm 0.0^{\mathrm{Db} a}$ & $4.55 \pm 0.05^{\mathrm{Cb} c}$ & $50.57 \pm 0.05^{\mathrm{Ab} b}$ & $0.00 \pm 0.0^{\mathrm{Dcd} d}$ & $4.55 \pm 0.01^{\mathrm{Ccd}}$ & & $19.89 \pm 0.04^{\mathrm{Bac}}$ & $3.98 \pm 0.01^{\mathrm{Ca} b}$ & $1.70 \pm 0.01 \mathrm{Cb} b$ & $21.59 \pm 0.09^{\mathrm{Ba} a}$ & $2.84 \pm 0.01^{\mathrm{Cc} b}$ & $6.82 \pm 0.01^{\mathrm{Cc} b}$ \\
\hline & 30 & $0.00 \pm 0.0^{\mathrm{Db} a}$ & $27 \pm 0.05^{\mathrm{Ccc}}$ & $77.84 \pm 0.09^{\mathrm{Aa} a}$ & $0.00 \pm 0.0^{\mathrm{Dcd} d}$ & & & $0.04^{\mathrm{Bbc}}$ & $0.00 \pm 0.0^{\mathrm{Dcc}}$ & $1.14 \pm 0.01^{\mathrm{Cb} b}$ & $0.00 \pm 0.0^{\mathrm{D} c c}$ & $13.64 \pm 0.01^{\mathrm{Bb}}$ & $10.80 \pm 0.01^{\mathrm{Bb} a}$ \\
\hline & 40 & $0.00 \pm 0.0^{\mathrm{Db} a}$ & $1.70 \pm 0.03^{\mathrm{Ccc}}$ & $29.55 \pm 0.08^{\mathrm{Ac} b}$ & $0.00 \pm 0.0^{\mathrm{Dcd} d}$ & $10.23 \pm 0.01^{\mathrm{Bb}}$ & $36.36 \pm$ & $32.39 \pm 0.02^{\mathrm{Aa} a}$ & $0.00 \pm 0.0^{\mathrm{Dcc}}$ & $6.25 \pm 0.01^{\mathrm{Cb} b}$ & $13.64 \pm 0.09^{\mathrm{Bba}}$ & $6.82 \pm 0.01^{\mathrm{Cc} b}$ & $20.45 \pm 0.01^{\mathrm{Ba} a}$ \\
\hline \multirow{6}{*}{2} & 0 & $00 \pm 0.0^{\mathrm{Db} a}$ & $29.55 \pm 0.05^{\mathrm{Aa} a}$ & $15.91 \pm 0.08^{\mathrm{Bac}}$ & $25.57 \pm 0.05^{\mathrm{Aac}}$ & $14.77 \pm 0.01^{\mathrm{Bac}}$ & 28.98 & $.02^{\mathrm{Aab}}$ & $0.00 \pm 0.0^{\mathrm{Dcc}}$ & $9.09 \pm 0.02^{\mathrm{Cb} b}$ & $6.25 \pm 0.01^{\mathrm{Ca} b}$ & $2.27 \pm 0.01^{\mathrm{Cac}}$ & $4.55 \pm 0.01^{\mathrm{Cb} b}$ \\
\hline & 5 & $0.57 \pm 0.01^{\mathrm{Da} a}$ & $23.30 \pm 0.05^{\mathrm{Aa} b}$ & $14.77 \pm 0.05^{\mathrm{Ba} b}$ & $18.18 \pm 0.01^{\mathrm{Bb} l}$ & b $13.64=$ & $34.09 \pm$ & $15.91 \pm 0.01^{\mathrm{Bb} c}$ & $0.00 \pm 0.0^{\mathrm{D} c c}$ & $5.68 \pm 0.05^{\mathrm{Cb} b}$ & $2.27 \pm 0.01^{\mathrm{Ca} b}$ & $0.00 \pm 0.0^{\mathrm{Db} d}$ & $15.91 \pm 0.01^{\mathrm{Ba} a}$ \\
\hline & 10 & $0.00 \pm 0.0^{\mathrm{Db} a}$ & $9.09 \pm 0.01^{\mathrm{Ccc}}$ & $9.09 \pm 0.08^{\mathrm{Cb} d}$ & $19.89 \pm 0.01^{\mathrm{Bb} l}$ & 15.34 & $21.59 \pm$ & $26.14 \pm 0.01^{\mathrm{Aa} a}$ & $0.00 \pm 0.0^{\mathrm{Dcc}}$ & $0.00 \pm 0.0^{\mathrm{Dcc}}$ & $6.25 \pm 0.01^{\mathrm{Ca} b}$ & $0.00 \pm 0.0^{\mathrm{Db} d}$ & $4.55 \pm 0.01^{\mathrm{Cb} b}$ \\
\hline & 20 & $0.00 \pm 0.0^{\mathrm{Db} a}$ & $6.82 \pm 0.01^{\mathrm{Bb} b}$ & $10.23 \pm 0.08^{\mathrm{Bac}}$ & $3.41 \pm 0.01^{\mathrm{Cc} c}$ & $3.41 \pm 0.01^{\mathrm{Cb} d}$ & $25.57 \pm$ & $15.91 \pm 0.01^{\mathrm{Bb} c}$ & $0.00 \pm 0.0^{\mathrm{D} c c}$ & $2.27 \pm 0.05^{\mathrm{Cb} b}$ & $4.55 \pm 0.01^{\mathrm{Ca} b}$ & $0.00 \pm 0.0^{\mathrm{Db} d}$ & $4.55 \pm 0.01^{\mathrm{Cb} b}$ \\
\hline & 30 & $0.00 \pm 0.0^{\mathrm{Db} a}$ & $10.80 \pm 0.03^{\mathrm{Cb} b}$ & $0.57 \pm 0.01^{\mathrm{Dc} f}$ & $4.55 \pm 0.02^{\mathrm{Ccc}}$ & $0.00 \pm 0.0^{\mathrm{Db} e}$ & $31.82 \pm 0.01^{\mathrm{Aa} a}$ & $35.23 \pm 0.01^{\mathrm{Aa} a}$ & $0.00 \pm 0.0^{\mathrm{Dcc}}$ & $0.00 \pm 0.0^{\mathrm{Bc} c}$ & $5.68 \pm 0.01^{\mathrm{Ca} b}$ & $0.00 \pm 0.0^{\mathrm{Db} d}$ & $13.07 \pm 0.01^{\mathrm{Ba} a}$ \\
\hline & 40 & $0.00 \pm 0.0^{\mathrm{Db} a}$ & $11.93 \pm 0.05^{\mathrm{Ba} a}$ & $0.00 \pm 0.0^{\mathrm{Da} f}$ & $5.68 \pm 0.05^{\mathrm{Ccc}}$ & $0.57 \pm 0.01^{\mathrm{Db} e}$ & $39.20 \pm 0.01^{\mathrm{A} z}$ & $22.73 \pm 0.01^{\mathrm{Bb} b}$ & $0.00 \pm 0.0^{\mathrm{Dcc}}$ & $13.64 \pm 0.05^{\mathrm{Ba}}$ & $6.82 \pm 0.01^{\mathrm{Ca} b}$ & $0.00 \pm 0.0^{\mathrm{Db} d}$ & $20.45 \pm 0.01^{\mathrm{Ba} a}$ \\
\hline \multirow{6}{*}{3} & 0 & $0.00 \pm 0.0^{\mathrm{Db} a}$ & $13.07 \pm 0.02^{\mathrm{Bab}}$ & $0.00 \pm 0.0^{\mathrm{Da} f}$ & $9.09 \pm 0.04^{\mathrm{Cb} c}$ & $10.80 \pm 0.01^{\mathrm{Ba}}$ & $22.73 \pm 0.02^{\mathrm{Ab} b}$ & $23.86 \pm 0.0^{\mathrm{Ab} b}$ & $0.00 \pm 0.0^{\mathrm{D} c c}$ & $0.00 \pm 0.0^{\mathrm{Dac}}$ & $1.14 \pm 0.01^{\mathrm{Cb} b}$ & $0.00 \pm 0.0^{\mathrm{Dad}}$ & $17.61 \pm 0.01^{\mathrm{Ba} a}$ \\
\hline & 5 & $0.00 \pm 0.0^{\mathrm{Db} a}$ & $14.20 \pm 0.02^{\mathrm{Ba} b}$ & $0.00 \pm 0.0^{\mathrm{Da} f}$ & $17.61 \pm 0.01^{\mathrm{Bal}}$ & $14.20 \pm 0.01^{\mathrm{Bac}}$ & $26.70 \pm 0.02^{\mathrm{Ab} b}$ & $11.93 \pm 0.01^{\mathrm{Bc}}$ & $0.00 \pm 0.0^{\mathrm{D} c c}$ & $0.00 \pm 0.0^{\mathrm{Dac}}$ & $6.82 \pm 0.01^{\mathrm{Ca} b}$ & $0.00 \pm 0.0^{\mathrm{Dad}}$ & $8.52 \pm 0.01^{\mathrm{Cc} b}$ \\
\hline & 10 & $0.00 \pm 0.0^{\mathrm{Db} a}$ & $3.41 \pm 0.03^{\mathrm{Cb} c}$ & $0.00 \pm 0.0^{\mathrm{Da} f}$ & $9.09 \pm 0.01^{\mathrm{Cb} b}$ & $14.77 \pm 0.01^{\mathrm{Bac}}$ & $29.55 \pm 0.02^{\mathrm{Ab} b}$ & $27.27 \pm 0.01^{\mathrm{Ab} b}$ & $0.00 \pm 0.0^{\mathrm{D} c c}$ & $0.00 \pm 0.0^{\mathrm{Dac}}$ & $2.27 \pm 0.01^{\mathrm{Cb} b}$ & $0.00 \pm 0.0^{\mathrm{Dad}}$ & $4.55 \pm 0.01^{\mathrm{Cc} b}$ \\
\hline & 20 & $0.00 \pm 0.0^{\mathrm{Db} a}$ & $8.52 \pm 0.04^{\mathrm{Cb} c}$ & $0.00 \pm 0.0^{\mathrm{Da} f}$ & $0.00 \pm 0.0^{\mathrm{Dcd} d}$ & $0.00 \pm 0.0^{\mathrm{Db} e}$ & $47.16 \pm 0.02^{\mathrm{Aa} a}$ & $15.34 \pm 0.01^{\mathrm{Bcc}}$ & $0.00 \pm 0.0^{\mathrm{D} c c}$ & $0.00 \pm 0.0^{\mathrm{Dac}}$ & $6.25 \pm 0.01^{\mathrm{Ca} b}$ & $0.00 \pm 0.0^{\mathrm{Dad}}$ & $13.64 \pm 0.01^{\mathrm{Bb} a}$ \\
\hline & 30 & $1.14 \pm 0.03^{\mathrm{Ca} b}$ & $3.41 \pm 0.02^{\mathrm{Ccc}}$ & $0.00 \pm 0.0^{\mathrm{Daf}}$ & $0.00 \pm 0.0^{\mathrm{Dcd} d}$ & $0.00 \pm 0.0^{\mathrm{Db} e}$ & $37.50 \pm 0.02^{\mathrm{Aa} a}$ & $34.09 \pm 0.01^{\mathrm{Ab} b}$ & $0.00 \pm 0.0^{\mathrm{D} c c}$ & $0.00 \pm 0.0^{\mathrm{Dac}}$ & $1.14 \pm 0.01^{\mathrm{Cb} b}$ & $0.00 \pm 0.0^{\mathrm{Dad}}$ & $22.16 \pm 0.01^{\mathrm{Ba} a}$ \\
\hline & 40 & $0.00 \pm 0.0^{\mathrm{Db} a}$ & $9.09 \pm 0.01^{\mathrm{Cb} c}$ & $0.00 \pm 0.0^{\mathrm{Da} f}$ & $0.00 \pm 0.0^{\mathrm{Dcd} d}$ & $0.00 \pm 0.0^{\mathrm{Db} e}$ & $29.55 \pm 0.02^{\mathrm{Bb} b}$ & $44.32 \pm 0.01^{\mathrm{Aa} a}$ & $2.27 \pm 0.01^{\mathrm{Cb} b}$ & $0.00 \pm 0.0^{\mathrm{Dac}}$ & $6.82 \pm 0.01^{\mathrm{Ca} b}$ & $0.00 \pm 0.0^{\mathrm{Dad}}$ & $25.00 \pm 0.01^{\mathrm{Ba} a}$ \\
\hline
\end{tabular}

** A, B , C , D ..: Capital letters show the difference between the months for each station and the difference between the months carrying different capital letters on the same line is statistically significant $(\mathrm{p}<0.05)$,

$\mathrm{a}, \mathrm{b}, \mathrm{c}, \mathrm{d} . .:$ Lower case letters show the difference between the depths for each station and the difference between the depths carrying different lower case letters in the same line is statistically significant $(\mathrm{p}<0.05)$,

$\mathrm{a}, \mathrm{b}, \mathrm{c}, \mathrm{d} . .$. Italic letters show the difference between stations for each station and the difference between the stations carrying different italic letters in the same line is statistically significant $(\mathrm{p}<0.05)$ 
Table 4. Change of Ammonia-Nitrogen $\left(\mathrm{NH}_{3}-\mathrm{N}\right)$ values depending on months, stations and depth on Tortum Lake (Mean $\left.\pm S D, m g L^{-1}\right)(n=4)$

\begin{tabular}{|c|c|c|c|c|c|c|c|c|c|c|c|c|c|}
\hline St & epth & Feb & Mar & Apr & May & Jun & Jul & Aug & Sep & Oct & Nov & Dec & Jan \\
\hline \multirow{6}{*}{1} & 0 & $0.07 \pm 0.011^{\mathrm{Cb} b}$ & $0.24 \pm 0.01^{\mathrm{Aa} a}$ & $.17 \pm 0.01^{\mathrm{Ba} a}$ & $20 \pm 0.01^{\mathrm{Aa} a}$ & $0.04 \pm 0.01^{\mathrm{Ca} a}$ & $0.10 \pm 0.01^{\mathrm{Ba} a}$ & $0.11 \pm 0.01^{\mathrm{Ba} a}$ & $0.10 \pm 0.01^{\mathrm{Ba} a}$ & $0.10 \pm 0.01^{\mathrm{Bc} b}$ & $0.13 \pm 0.01^{\mathrm{Bb} a}$ & $0.13 \pm 0.01^{\mathrm{Ba} a}$ & $0.00 \pm 0.0^{\mathrm{Dad}}$ \\
\hline & 5 & $0.08 \pm 0.011^{\mathrm{Cb} b}$ & $0.24 \pm 0.01^{\mathrm{Aa} a}$ & $0.17 \pm 0.01^{\text {Ba } a}$ & $0.17 \pm 0.01^{\mathrm{Ab} a}$ & $0.05 \pm 0.01$ Сa $a$ & $0.08 \pm 0.01^{\mathrm{Cb} a}$ & $0.12 \pm 0.01^{\mathrm{Ba} a}$ & $0.12 \pm 0.01^{\mathrm{Ba} a}$ & $0.08 \pm 0.01^{\mathrm{Cd} c}$ & $0.12 \pm 0.01^{\mathrm{Bb} a}$ & $0.13 \pm 0.01 \mathrm{Bac}$ & $0.00 \pm 0.0 \mathrm{Dad}$ \\
\hline & 10 & & $.25 \pm 0.01^{\mathrm{Aa} a}$ & $0.18 \pm 0.01^{\mathrm{Ba} a}$ & $0.14 \pm 0.01^{\mathrm{Bb} a}$ & $0.05 \pm 0.01^{\mathrm{Ca} a}$ & $0.11 \pm 0.01^{\mathrm{Ba} a}$ & $0.10 \pm 0.01^{\mathrm{Ba} a}$ & & $0.21 \pm 0.01^{\mathrm{Aa} a}$ & $0.11 \pm 0.01^{\mathrm{Bb} a}$ & $0.15 \pm 0.01 \mathrm{Bac}$ & $0.00 \pm 0.0 \mathrm{Dad}$ \\
\hline & 20 & & $24 \pm 0.01^{\mathrm{Aa} a}$ & $0.17 \pm 0.01^{\mathrm{Ba} a}$ & $0.16 \pm 0.01^{\mathrm{Bb} a}$ & $0.09 \pm 0.01^{\mathrm{Ca} a}$ & $0.12 \pm 0.01^{\mathrm{Ba} a}$ & $0.13 \pm 0.01^{\mathrm{Ba} a}$ & & $0.16 \pm 0.01^{\mathrm{Bb} b}$ & $0.12 \pm 0.01^{\mathrm{Bb} a}$ & $0.12 \pm 0.01 \mathrm{Bac}$ & $0.00 \pm 0.0 \mathrm{Dad}$ \\
\hline & 30 & & $25 \pm 0.01^{\mathrm{Aa} a}$ & $.01^{\mathrm{Ba} a}$ & & $08 \pm 0.01^{\mathrm{Ca} a}$ & $0.12 \pm 0.01^{\mathrm{Ba} a}$ & & & & $0.16 \pm 0.01^{\mathrm{Bb} a}$ & $0.16 \pm 0.01^{\mathrm{Bac}}$ & $0.00 \pm 0.0^{\mathrm{Dad} d}$ \\
\hline & 40 & & $01^{\mathrm{Aa} a}$ & $0.18 \pm 0$ & $01^{\mathrm{Bb} a}$ & .01 Сa $a$ & $01^{\mathrm{Ba} a}$ & $0.13 \pm 0.01^{\mathrm{Ba} a}$ & $0.10 \pm 0.01^{\mathrm{Ba} a}$ & $0.14 \pm 0.01^{\mathrm{Bc} b}$ & $0.13 \pm 0.01^{\mathrm{Bb} a}$ & $0.12 \pm 0.01^{\mathrm{Bac}}$ & $0.00 \pm 0.0^{\mathrm{Dad} d}$ \\
\hline \multirow{6}{*}{2} & 0 & & $23 \pm 0.01^{\mathrm{Aa} a}$ & $\mathrm{Ba} a$ & Ba $a$ & $0.06 \pm 0$ & $0.15 \pm 0$ & $0.09 \pm 0.01^{\mathrm{Cb} a}$ & $0.09 \pm$ & $0.13 \pm$ & $0.14 \pm$ & $0.15 \pm 0.01^{\mathrm{Bac}}$ & $0.09 \pm 0$. \\
\hline & 5 & $0.08 \pm 0.01^{\mathrm{Cb} b}$ & $24 \pm 0.01^{\mathrm{Aa} a}$ & $0.18 \pm 0.01^{\mathrm{Ba} a}$ & $0.19 \pm 0.01^{\mathrm{Ba} a}$ & $0.07 \pm 0.01^{\mathrm{Ca} a}$ & $0.11 \pm 0.01^{\mathrm{Ba} a}$ & $0.12 \pm 0.01^{\mathrm{Ba} a}$ & $0.15 \pm 0.01^{\mathrm{Ba} a}$ & $0.11 \pm 0.01^{\mathrm{Ba} b}$ & $0.14 \pm 0.01^{\mathrm{Ba} a}$ & $0.18 \pm 0.01^{\mathrm{Bac}}$ & $0.13 \pm 0.02^{\mathrm{Bb} b}$ \\
\hline & 10 & $0.08 \pm 0.01^{\mathrm{Cb} b}$ & $25 \pm 0.01^{\mathrm{Aa} a}$ & $0.14 \pm 0.01^{\mathrm{Ba} a}$ & $0.19 \pm 0.01^{\text {Ва } a}$ & $0.07 \pm 0.01$ Сa $a$ & $0.13 \pm 0.01^{\mathrm{Ba} a}$ & $0.12 \pm 0.01^{\mathrm{Ba} a}$ & $0.11 \pm 0.01^{\mathrm{Ba} a}$ & $0.15 \pm 0.01^{\mathrm{Ba} b}$ & $0.12 \pm 0.01^{\mathrm{Ba} a}$ & $0.16 \pm 0.01 \mathrm{Bac}$ & $0.34 \pm 0.02^{\mathrm{Aa} a}$ \\
\hline & 20 & $0.09 \pm 0.01^{\mathrm{Cb} b}$ & $.21 \pm 0.01^{\mathrm{Aa} a}$ & $0.18 \pm 0.01^{\mathrm{Ba} a}$ & $0.16 \pm 0.01^{\mathrm{Ba} a}$ & $0.08 \pm 0.01$ Сa $a$ & $0.11 \pm 0.01^{\mathrm{Ba} a}$ & $0.12 \pm 0.01^{\mathrm{Ba} a}$ & $0.11 \pm 0.01^{\mathrm{Ba} a}$ & $0.13 \pm 0.01^{\mathrm{Ba} b}$ & $0.13 \pm 0.01^{\mathrm{Ba} a}$ & $0.16 \pm 0.01 \mathrm{Bac}$ & $0.09 \pm 0.01^{\mathrm{Cc} c}$ \\
\hline & 30 & $0.07 \pm 0.01^{\mathrm{Cb} b}$ & $0.23 \pm 0.01^{\mathrm{Aa} a}$ & $0.18 \pm 0.01^{\text {Ba } a}$ & $0.18 \pm 0.01^{\mathrm{Ba} a}$ & $0.07 \pm 0.01^{\mathrm{Ca} a}$ & $0.13 \pm 0.01^{\mathrm{Ba} a}$ & $0.12 \pm 0.01^{\mathrm{Ca} a}$ & $0.15 \pm 0.01^{\mathrm{Ba} a}$ & $0.11 \pm 0.01^{\mathrm{Ba} b}$ & $0.13 \pm 0.01^{\mathrm{Ba} a}$ & $0.18 \pm 0.01^{\mathrm{Bac}}$ & $0.10 \pm 0.01^{\mathrm{Bb} b}$ \\
\hline & 40 & $0.07 \pm 0.01^{\mathrm{Da} a}$ & $0.24 \pm 0.01^{\mathrm{Ba} a}$ & $0.17 \pm 0.01^{\mathrm{Ca} a}$ & $0.14 \pm 0.01^{\text {С } a}$ & $0.08 \pm 0.01^{\mathrm{Da} a}$ & $0.11 \pm 0.01^{\text {Са } a}$ & $0.12 \pm 0.01^{\mathrm{Ca} a}$ & $0.12 \pm 0.01^{\mathrm{Ca} a}$ & $0.10 \pm 0.01^{\mathrm{Ca} b}$ & $0.14 \pm 0.01^{\mathrm{Ba} a}$ & $0.11 \pm 0.01^{\mathrm{Ca} a}$ & $0.43 \pm 0.02^{\mathrm{Aa} a}$ \\
\hline & 0 & $0.06 \pm 0.01^{\mathrm{Cb} b}$ & $0.18 \pm 0.01^{\mathrm{Bb} b}$ & $0.10 \pm 0.01^{\mathrm{Ba} a}$ & $0.14 \pm 0.01^{\mathrm{Ba} a}$ & $0.07 \pm 0.01$ Ca $a$ & $0.13 \pm 0.01^{\mathrm{Ba} a}$ & $0.00 \pm 0.0^{\mathrm{Db} b}$ & $0.17 \pm 0.01^{\mathrm{Ba} a}$ & $0.10 \pm 0.01^{\mathrm{Ba} b}$ & $0.14 \pm 0.01$ Ca $a$ & $0.13 \pm 0.01^{\mathrm{Ba} a}$ & $0.43 \pm 0.01^{\mathrm{Aa} a}$ \\
\hline & 5 & $0.09 \pm 0.01^{\mathrm{Cb} b}$ & $0.19 \pm 0.01^{\mathrm{Bb} b}$ & $0.15 \pm 0.01^{\mathrm{Ba} a}$ & $0.18 \pm 0.01^{\text {Ва } a}$ & $0.06 \pm 0.01^{\mathrm{Ca} a}$ & $0.11 \pm 0.01^{\mathrm{Ba} a}$ & $0.11 \pm 0.01^{\mathrm{Ba} a}$ & $0.17 \pm 0.01^{\mathrm{Ba} a}$ & $0.12 \pm 0.01^{\mathrm{Db} c}$ & $0.15 \pm 0.01^{\mathrm{Ba} a}$ & $0.14 \pm 0.01^{\mathrm{Ba} a}$ & $0.45 \pm 0.02^{\mathrm{Aa} a}$ \\
\hline & 10 & $0.10 \pm 0.01^{\mathrm{Cb} b}$ & $0.22 \pm 0.01^{\mathrm{Ba} a}$ & $0.12 \pm 0.01^{\mathrm{Da} a}$ & $0.21 \pm 0.01^{\mathrm{Ba} a}$ & $0.05 \pm 0.01^{\mathrm{Da} a}$ & $0.11 \pm 0.01^{\mathrm{Ca} a}$ & $0.11 \pm 0.01^{\mathrm{Ca} a}$ & $0.16 \pm 0.01^{\text {Сa } a}$ & $0.09 \pm 0.01^{\mathrm{Db} c}$ & $0.13 \pm 0.01^{\mathrm{Ba} a}$ & $0.14 \pm 0.01^{\mathrm{Ca} a}$ & $0.40 \pm 0.02^{\mathrm{Aa} a}$ \\
\hline & 20 & $0.11 \pm 0.01^{\mathrm{Cb} b}$ & $0.20 \pm 0.01^{\mathrm{Ba} a}$ & $0.16 \pm 0.01^{\mathrm{Ba} a}$ & $0.17 \pm 0.01^{\mathrm{Ba} a}$ & $0.08 \pm 0.01^{\mathrm{Ca} a}$ & $0.09 \pm 0.01^{\mathrm{Cb} a}$ & $0.12 \pm 0.01^{\mathrm{Ba} a}$ & $0.17 \pm 0.01^{\mathrm{Ba} a}$ & $0.14 \pm 0.01^{\mathrm{Ba} b}$ & $0.13 \pm 0.01^{\mathrm{Ca} a}$ & $0.15 \pm 0.01^{\mathrm{Ba} a}$ & $0.42 \pm 0.02^{\mathrm{Aa} a}$ \\
\hline & 30 & $0.09 \pm 0.01^{\mathrm{Cb} b}$ & $0.27 \pm 0.01^{\mathrm{Ba} a}$ & $0.17 \pm 0.01^{\mathrm{Ba} a}$ & $0.20 \pm 0.01^{\text {Ва } a}$ & $0.07 \pm 0.01^{\mathrm{Ca} a}$ & $0.11 \pm 0.01^{\mathrm{Ba} a}$ & $0.12 \pm 0.01^{\mathrm{Ba} a}$ & $0.17 \pm 0.01^{\mathrm{Ba} a}$ & $0.12 \pm 0.01^{\mathrm{Ba} b}$ & $0.12 \pm 0.01^{\mathrm{Ba} a}$ & $0.14 \pm 0.01^{\mathrm{Ba} a}$ & $0.39 \pm 0.02^{\mathrm{Aa} a}$ \\
\hline & 40 & $0.08 \pm 0.01^{\mathrm{Db} b}$ & $0.24 \pm 0.01^{\mathrm{Ba} a}$ & $0.15 \pm 0.01^{\mathrm{Ca} a}$ & $0.19 \pm 0.01^{\mathrm{Cb} a}$ & $0.07 \pm 0.01^{\mathrm{Da} a}$ & $0.07 \pm 0.01^{\mathrm{Db} a}$ & $0.12 \pm 0.01^{\mathrm{Ca} a}$ & $0.16 \pm 0.01^{\mathrm{Ca} a}$ & $0.12 \pm 0.01^{\mathrm{Ca} b}$ & $0.13 \pm 0.01^{\mathrm{Ca} a}$ & $0.16 \pm 0.01^{\mathrm{Ca} a}$ & $0.42 \pm 0.02 \mathrm{~A}^{\mathrm{ac}}$ \\
\hline
\end{tabular}

** A, B , C, D ..: Capital letters show the difference between the months for each station and the difference between the months carrying different capital letters on the same line is statistically significant $(\mathrm{p}<0.05)$,

$\mathrm{a}, \mathrm{b}, \mathrm{c}, \mathrm{d} . .:$ Lower case letters show the difference between the depths for each station and the difference between the depths carrying different lower case letters in the same line is statistically significant $(\mathrm{p}<0.05)$,

$\mathrm{a}, \mathrm{b}, \mathrm{c}, \mathrm{d}$..: Italic letters show the difference between stations for each station and the difference between the stations carrying different italic letters in the same line is statistically significant $(\mathrm{p}<0.05)$ 
When the $\mathrm{NO}_{3}-\mathrm{N}$ values were examined according to different months and stations, the lowest mean value at the first station was $0.02 \pm 0.01 \mathrm{mgL}^{-1}$ in January, while the highest value was $6.06 \pm 0.05 \mathrm{mgL}^{-1}$ in September. The lowest mean value at the second station was $0.01 \pm 0.01 \mathrm{mgL}^{-1}$ on January, while the highest value was $5.59 \pm 0.02 \mathrm{mgL}^{-1}$ in September. The lowest mean value at the third station was $0.0 \pm 0.0 \mathrm{mgL}^{-1}$ in August and January, while the highest value was $9.45 \pm 0.05 \mathrm{mgL}^{-1}$ in September. The mean values of $\mathrm{NO}_{3}-\mathrm{N}$ at the first, second, and third stations were $1.05 \pm 0.02 \mathrm{mgL}^{-1}$, $0.99 \pm 0.05 \mathrm{mgL}^{-1}$, and $1.27 \pm 0.03 \mathrm{mgL}^{-1}$, respectively (Table 5).

$\mathrm{NO}_{2}-\mathrm{N}$ values were lowest in February, March, and January for all stations and were highest in December. In March, July, and January, the second and third stations $\mathrm{NO}_{2}-\mathrm{N}$ values for all depths were $0.0 \pm 0.0 \mathrm{mgL}^{-1}$. During the same period at the first station, a concentration of $0.38 \pm 0.01 \mathrm{mgL}^{-1}$ was measured, at the surface only. The largest $\mathrm{NO}_{2}-\mathrm{N}$ values at the first, second, and third stations were detected as $4.38 \pm 0.01 \mathrm{mgL}^{-1}$, $4.69 \pm 0.01 \mathrm{mgL}^{-1}$, and $5.63 \pm 0.01 \mathrm{mgL}^{-1}$, respectively (Table 6).

\section{External phosphorus loading in Lake Tortum}

The land phosphorus load was calculated by the multiplication of phosphorus export coefficient $\left(\mathrm{kg} \mathrm{yr}^{-1}\right)$ and catchment area $\left(\mathrm{km}^{2}\right)$. Tortum Stream continuously supplies water to Lake Tortum from its catchment area of $16.534 \mathrm{~km}^{2}$. The phosphorus export coefficient (Ep) was calculated to be $0.07934 \mathrm{~kg} \mathrm{~km}^{-2}$ according to Kirchner and Dillon (1975) methods. The morphometric and hydrological parameters of Lake Tortum have been supplyed from DSI (Table 7).

The land phosphorus load was found to be $1312 \mathrm{~kg} \mathrm{yr}^{-1}$ (Equation 1), while the atmospheric loading (AL) is $31 \mathrm{~kg} \mathrm{yr}^{-1}$ (Equation 2). The natural phosphorus load is $1343 \mathrm{~kg} \mathrm{yr}^{-1}$ (Equation 3), whereas the domestic phosphorus load is $9707 \mathrm{~kg} \mathrm{yr}^{-1}$ (Equation 4). The external TP loading to the Lake Tortum is $11050 \mathrm{~kg} \mathrm{yr}^{-1}$. The TP load coeficient was computed to be $1.71 \mathrm{~g} \cdot \mathrm{m}^{-2} \cdot \mathrm{yr}^{-1}$ (Equation 6). According to the formulas developed by Chapra and Tarapchak (1976) and Vollenweider (1976) from the calculated phosphorus loadings of Lake Tortum measured in 2017.

$$
\begin{aligned}
\mathrm{L}_{\text {critical }}= & 10 \mathrm{x} 74.42(1+0.34641) \\
= & 1002 \mathrm{mg} \cdot \mathrm{m}^{-2} \cdot \mathrm{yr}^{-1} \\
& \sim 1.002 \mathrm{~g} \cdot \mathrm{m}^{-2} \cdot \mathrm{yr}^{-1} \\
\mathrm{~L}_{\text {permit }} & =0.011(74.42+12.4) \\
& =0.96 \mathrm{~g} \cdot \mathrm{m}^{-2} \cdot \mathrm{yr}^{-1} \\
\mathrm{~L}_{\text {critical }} & =0.025(74.42+12.4) \\
& =2.17 \mathrm{~g} \cdot \mathrm{m}^{-2} \cdot \mathrm{yr}^{-1} \\
\mathrm{~L}_{\text {critical }}=0.0055(0.04)^{0.69}(74.42+12.4) & \\
& =0.05 \mathrm{~g} \cdot \mathrm{m}^{-2} \cdot \mathrm{yr}^{-1}
\end{aligned}
$$


Table 5. Change of Nitrate-Nitrogen $\left(\mathrm{NO}_{3}-\mathrm{N}\right)$ values depending on months, stations and depth on Tortum Lake (Mean $\left.\pm S D, m g L^{-1}\right)(n=4)$

\begin{tabular}{|c|c|c|c|c|c|c|c|c|c|c|c|c|c|}
\hline St & Depth & Feb & Mar & Apr & May & Jun & Jul & Aug & Sep & Oct & Nov & Dec & Jan \\
\hline \multirow{6}{*}{1} & 0 & $0.09 \pm 0.01^{\mathrm{Cb} b}$ & $0.05 \pm 0.01^{\mathrm{Cb} c}$ & $0.02 \pm 0.01^{\mathrm{Cad}}$ & $.14 \pm 0.01^{\mathrm{Bcd}}$ & $.27 \pm 0.01^{\mathrm{Bb} c}$ & $0.26 \pm 0.01^{\mathrm{Bb} d}$ & $0.12 \pm 0.01^{\mathrm{Bb} b}$ & $1.04 \pm 0.01^{\mathrm{Ac} b}$ & $6.22 \pm 0.01^{\text {Аa } a}$ & $2.49 \pm 0.01^{\mathrm{Ab} b}$ & $0.09 \pm 0.01^{\mathrm{Cb} b}$ & $0.03 \pm 0.01^{\mathrm{A} a}$ \\
\hline & 5 & $0.10 \pm 0.02 \mathrm{~B}^{\mathrm{ac}}$ & $00 \pm 0.01^{\mathrm{Dc} d}$ & $0.02 \pm 0.01^{\mathrm{Cad} d}$ & $0.32 \pm 0.01^{\mathrm{Bb} c}$ & $0.15 \pm 0.01^{\mathrm{Bcd}}$ & $0.16 \pm 0.01_{\mathrm{Bc} d}$ & $0.00 \pm 0.0^{\mathrm{Dcd}}$ & $1.59 \pm 0.05^{\mathrm{Ac} b}$ & $2.77 \pm 0.01^{\mathrm{Ab} b}$ & $3.53 \pm 0.01^{\mathrm{Aa} b}$ & $0.08 \pm 0.01^{\mathrm{Cb} b}$ & $0.00 \pm 0.01^{\mathrm{Db} b}$ \\
\hline & 10 & $0.10 \pm 0.02^{\mathrm{Ba} a}$ & $0.06 \pm 0.01^{\mathrm{Cb} c}$ & $0.03 \pm 0.01 \mathrm{Cad}$ & $0.47 \pm 0.01^{\mathrm{Bb} b}$ & $0.20 \pm 0.01^{\mathrm{Bcc}}$ & $0.17 \pm 0.01^{\mathrm{Bc} d}$ & $0.00 \pm 0.0^{\mathrm{Dc} d}$ & $1.87 \pm 0.05^{\mathrm{Acb}}$ & $2.70 \pm 0.01^{\mathrm{Ab} b}$ & $3.32 \pm 0.01^{\mathrm{Aa} b}$ & $0.08 \pm 0.01^{\mathrm{Cb} b}$ & $0.03 \pm 0.01^{\mathrm{Ca} a}$ \\
\hline & 20 & $0.07 \pm 0.02^{\mathrm{Cb} b}$ & $19 \pm 0.01^{\mathrm{Ba} b}$ & $0.06 \pm 0.01^{\mathrm{Cad} d}$ & $0.56 \pm 0.01^{\mathrm{Ba} b}$ & $0.19 \pm 0.01^{\mathrm{Bc} d}$ & $0.07 \pm 0.01^{\mathrm{Cd} e}$ & $0.00 \pm 0.0^{\mathrm{D} c d}$ & $15.69 \pm 0.05^{\mathrm{Aa} a}$ & $4.84 \pm 0.01^{\mathrm{Ba} b}$ & $4.42 \pm 0.01^{\mathrm{Ba} a}$ & $0.09 \pm 0.01^{\mathrm{Cb} b}$ & $0.02 \pm 0.01^{\mathrm{Ca} a}$ \\
\hline & 30 & $0.10 \pm 0.02^{\mathrm{Ba} a}$ & $15 \pm 0.01^{\mathrm{Ba} b}$ & $0.06 \pm 0.01^{\mathrm{Cad} d}$ & $0.48 \pm 0.01^{\mathrm{Bb} b}$ & $0.31 \pm 0.01^{\mathrm{Bb} b}$ & $0.13 \pm 0.01^{\mathrm{Ba} b}$ & $0.00 \pm 0.0^{\mathrm{Dc} d}$ & $3.25 \pm 0.01^{\mathrm{Bb} b}$ & $0.00 \pm 0.0^{\mathrm{Dcc}}$ & $0.62 \pm 0.01^{\mathrm{Bcc}}$ & $0.31 \pm 0.02^{\mathrm{Ba} a}$ & $0.02 \pm 0.01^{\mathrm{Ca} a}$ \\
\hline & 40 & $0.09 \pm 0.01^{\mathrm{Cb} b}$ & $0.07 \pm 0.01^{\mathrm{cb} c}$ & $0.04 \pm 0.01^{\mathrm{Cad}}$ & $0.44 \pm 0.01^{\mathrm{Bb} b}$ & $0.42 \pm 0.01^{\mathrm{Ba} a}$ & $0.68 \pm 0.01^{\mathrm{Ba} b}$ & $0.26 \pm 0.01^{\mathrm{Ba} a}$ & $12.93 \pm 0.05^{\mathrm{Aa} a}$ & $0.00 \pm 0.0^{\mathrm{D} c c}$ & $0.28 \pm 0.01^{\mathrm{Bcc}}$ & $0.04 \pm 0.01^{\mathrm{Cb} b}$ & $0.02 \pm 0.01^{\mathrm{Ca} a}$ \\
\hline \multirow{6}{*}{2} & 0 & & $22 \pm 0.02^{\mathrm{Ba} a}$ & $0.16 \pm 0.01^{\mathrm{Bcc}}$ & $0.37 \pm 0.01^{\mathrm{Bcc}}$ & $.29 \pm 0.01^{\mathrm{Bb} c}$ & $0.38 \pm 0.01^{\mathrm{Bcc}}$ & $0.15 \pm 0.01^{\mathrm{Ba} b}$ & $1.87 \pm 0.05^{\mathrm{Acb}}$ & $4.36 \pm 0.01^{\text {Aab }}$ & $3.39 \pm 0.01^{\mathrm{Aa} b}$ & $0.20 \pm 0.02^{\mathrm{Ba} a}$ & $0.01 \pm 0.01^{\mathrm{Ca} a}$ \\
\hline & 5 & $0.24 \pm 0.03^{\mathrm{Ba} a}$ & $0.14 \pm 0.01^{\mathrm{Bb} b}$ & $0.21 \pm 0.03^{\mathrm{Bb} b}$ & $0.53 \pm 0.01^{\mathrm{Ba} b}$ & $0.31 \pm 0.01^{\mathrm{Ba} b}$ & $0.42 \pm 0.01^{\mathrm{Bcc}}$ & $0.00 \pm 0.0^{\mathrm{Db} d}$ & $4.08 \pm 0.01^{\mathrm{Ab} b}$ & $4.42 \pm 0.01^{\mathrm{Aa} b}$ & $1.38 \pm 0.01^{\mathrm{Ab} b}$ & $0.07 \pm 0.01^{\mathrm{Cb} b}$ & $0.02 \pm 0.01^{\mathrm{Ca} a}$ \\
\hline & 10 & $0.20 \pm 0.03^{\mathrm{Bb} a}$ & $0.19 \pm 0.01^{\mathrm{Ba} a}$ & $0.23 \pm 0.02^{\mathrm{Ba} a}$ & $0.48 \pm 0.01^{\mathrm{Bb} b}$ & $0.23 \pm 0.01^{\mathrm{Bb} c}$ & $0.23 \pm 0.01^{\mathrm{Bd} d}$ & $0.00 \pm 0.0^{\mathrm{Db} d}$ & $3.53 \pm 0.01^{\mathrm{Ab} b}$ & $4.91 \pm 0.01^{\mathrm{Aa} b}$ & $3.94 \pm 0.01^{\mathrm{Aa} b}$ & $0.05 \pm 0.02^{\mathrm{Ba} a}$ & $0.00 \pm 0.0^{\mathrm{Db} b}$ \\
\hline & 20 & $0.23 \pm 0.03^{\mathrm{Ba} a}$ & $0.22 \pm 0.02^{\mathrm{Bb} b}$ & $0.23 \pm 0.02^{\mathrm{Ba} a}$ & $0.46 \pm 0.01^{\mathrm{Bb} b}$ & $0.26 \pm 0.01^{\mathrm{Bb} c}$ & $0.83 \pm 0.01^{\mathrm{Ba} a}$ & $0.00 \pm 0.0^{\mathrm{Db} d}$ & $4.22 \pm 0.01^{\mathrm{Ab} b}$ & $1.59 \pm 0.01^{\mathrm{Aa} b}$ & $0.83 \pm 0.01^{\mathrm{Bc} c}$ & $0.33 \pm 0.01^{\mathrm{Cb} b}$ & $0.00 \pm 0.0^{\mathrm{Cb} b}$ \\
\hline & 30 & $0.19 \pm 0.01^{\mathrm{Ba} a}$ & $0.15 \pm 0.02^{\mathrm{Bb} b}$ & $0.17 \pm 0.02^{\mathrm{Bb} b}$ & $0.43 \pm 0.01^{\mathrm{Bb} b}$ & $0.29 \pm 0.01^{\mathrm{Bb} c}$ & $0.27 \pm 0.01^{\mathrm{Bd} c}$ & $0.00 \pm 0.0^{\mathrm{Db} d}$ & $3.87 \pm 0.01^{\mathrm{Ab} b}$ & $0.28 \pm 0.01^{\mathrm{Bc} b}$ & $0.00 \pm 0.0^{\mathrm{Dd} d}$ & $0.06 \pm 0.01^{\mathrm{Cb} b}$ & $0.00 \pm 0.0^{\mathrm{Db} b}$ \\
\hline & 40 & $0.24 \pm 0.03^{\mathrm{Ba} a}$ & $0.11 \pm 0.01^{\mathrm{Ba} b}$ & $0.31 \pm 0.03^{\mathrm{Ba} a}$ & $0.68 \pm 0.01^{\mathrm{Bb} b}$ & $0.35 \pm 0.01^{\mathrm{Ba} b}$ & $0.79 \pm 0.01^{\mathrm{Bb} a}$ & $0.00 \pm 0.0^{\mathrm{Db} d}$ & $15.97 \pm 0.05^{\mathrm{Ab} a}$ & $0.00 \pm 0.0^{\mathrm{Ccc}}$ & $0.41 \pm 0.01^{\mathrm{Bcc}}$ & $0.28 \pm 0.01^{\mathrm{Ba} a}$ & $0.00 \pm 0.0^{\mathrm{Cb} b}$ \\
\hline \multirow{6}{*}{3} & 0 & $0.15 \pm 0.03^{\mathrm{Bb} a}$ & $0.19 \pm 0.02^{\mathrm{Dcd}}$ & $0.20 \pm 0.03^{\mathrm{Bb} b}$ & $0.60 \pm 0.01^{\mathrm{Bb} b}$ & $0.16 \pm 0.01^{\mathrm{Bb} d}$ & $0.39 \pm 0.01^{\mathrm{Bac}}$ & $0.00 \pm 0.0^{\mathrm{Cb} d}$ & $3.25 \pm 0.01^{\mathrm{Ac} b}$ & $2.97 \pm 0.01^{\mathrm{Ab} b}$ & $1.45 \pm 0.01^{\mathrm{Ab} b}$ & $0.40 \pm 0.03^{\mathrm{Ba} a}$ & $0.00 \pm 0.0^{\mathrm{Ca} b}$ \\
\hline & 5 & $0.16 \pm 0.03^{\mathrm{Ba} a}$ & $0.00 \pm 0.0^{\mathrm{Cb} c}$ & $0.18 \pm 0.03^{\mathrm{Bb} b}$ & $0.54 \pm 0.01^{\mathrm{Bb} b}$ & $0.33 \pm 0.01^{\mathrm{Ba} b}$ & $0.38 \pm 0.01^{\mathrm{Bac}}$ & $0.01 \pm 0.01^{\mathrm{Cac}}$ & $8.85 \pm 0.02^{\mathrm{Ab} b}$ & $6.64 \pm 0.01^{\mathrm{Aa} a}$ & $2.90 \pm 0.01^{\text {Aab }}$ & $0.05 \pm 0.01^{\mathrm{Cb} b}$ & $0.00 \pm 0.0^{\mathrm{Da} b}$ \\
\hline & 10 & $0.16 \pm 0.02^{\mathrm{Ba} a}$ & $0.03 \pm 0.01^{\mathrm{Db} c}$ & $0.20 \pm 0.03^{\mathrm{Bb} b}$ & $0.37 \pm 0.01^{\mathrm{Bcc}}$ & $0.31 \pm 0.01^{\mathrm{Ba} b}$ & $0.18 \pm 0.01^{\mathrm{Bb} d}$ & $0.00 \pm 0.0^{\mathrm{Db} d}$ & $5.05 \pm 0.03^{\mathrm{Ab} b}$ & $3.87 \pm 0.01^{\mathrm{Ab} b}$ & $2.70 \pm 0.01^{\text {Aab }}$ & $0.05 \pm 0.01^{\mathrm{Cb} b}$ & $0.00 \pm 0.0^{\mathrm{Da} b}$ \\
\hline & 20 & $0.11 \pm 0.01^{\mathrm{Cb} a}$ & $0.05 \pm 0.01^{\mathrm{Eb} c}$ & $0.27 \pm 0.02^{\mathrm{Ca} b}$ & $0.42 \pm 0.01^{\mathrm{Bc} b}$ & $0.30 \pm 0.01^{\mathrm{Ca} b}$ & $0.26 \pm 0.01^{\mathrm{Cb} d}$ & $0.00 \pm 0.0^{\mathrm{Eb} d}$ & $4.98 \pm 0.03^{\mathrm{Ab} b}$ & $0.83 \pm 0.01^{\mathrm{Bc} b}$ & $0.28 \pm 0.01^{\mathrm{Cc} c}$ & $0.06 \pm 0.01^{\mathrm{Db} b}$ & $0.00 \pm 0.0^{\mathrm{E} a b}$ \\
\hline & 30 & $0.11 \pm 0.01^{\mathrm{Db} a}$ & $0.08 \pm 0.01^{\mathrm{Gc} d}$ & $0.29 \pm 0.01^{\mathrm{Da} b}$ & $0.96 \pm 0.01^{\mathrm{Ca} a}$ & $0.33 \pm 0.01^{\mathrm{Da} b}$ & $0.41 \pm 0.01^{\mathrm{Dac}}$ & $0.00 \pm 0.0^{\mathrm{Eb} d}$ & $16.18 \pm 0.02^{\mathrm{Aa} a}$ & $0.00 \pm 0.0^{\mathrm{Fd} c}$ & $1.59 \pm 0.01^{\mathrm{bb} b}$ & $0.03 \pm 0.01^{\mathrm{Db} b}$ & $0.00 \pm 0.0^{\mathrm{Fa} b}$ \\
\hline & 40 & $0.13 \pm 0.03^{\mathrm{Eb} a}$ & $0.00 \pm 0.0^{\mathrm{Gcd} d}$ & $0.29 \pm 0.01^{\mathrm{Da} b}$ & $0.68 \pm 0.01^{\mathrm{Cb} b}$ & $0.37 \pm 0.01^{\mathrm{Da} b}$ & $0.32 \pm 0.01^{\mathrm{Dac}}$ & $0.00 \pm 0.0^{\mathrm{Gb} d}$ & $18.39 \pm 0.05^{\mathrm{Aa} a}$ & $0.90 \pm 0.01^{\mathrm{Bc} b}$ & $0.00 \pm 0.0^{\mathrm{gd} b}$ & $0.07 \pm 0.01^{\mathrm{Fb} b}$ & $0.00 \pm 0.0^{\mathrm{G} a b}$ \\
\hline
\end{tabular}

** A, B , C , D ..: Capital letters show the difference between the months for each station and the difference between the months carrying different capital letters on the same line is statistically significant $(\mathrm{p}<0.05)$,

$\mathrm{a}, \mathrm{b}, \mathrm{c}, \mathrm{d} . .:$ Lower case letters show the difference between the depths for each station and the difference between the depths carrying different lower case letters in the same line is statistically significant $(\mathrm{p}<0.05)$,

$\mathrm{a}, \mathrm{b}, \mathrm{c}, \mathrm{d} . .$. Italic letters show the difference between stations for each station and the difference between the stations carrying different italic letters in the same line is statistically significant $(\mathrm{p}<0.05)$ 
Table 6. Change of Nitrite-Nitrogen $\left(\mathrm{NO}_{2}-\mathrm{N}\right)$ values depending on months, stations and depth on Tortum Lake (Mean $\left.\pm S D, m g L^{-1}\right)(n=4)$

\begin{tabular}{|c|c|c|c|c|c|c|c|c|c|c|c|c|c|}
\hline St & Depth & Feb & Mar & Apr & May & Jun & Jul & Aug & Sep & Oct & Nov & Dec & Jan \\
\hline \multirow{6}{*}{1} & 0 & $0.00 \pm 0.0^{\mathrm{Dc} c^{*}}$ & $38 \pm 0.01^{\mathrm{Ba} a}$ & $0.55 \pm 0.01^{\mathrm{Bb} c}$ & $1.27 \pm 0.01^{\mathrm{Aa} b}$ & $0.03 \pm 0.01^{\mathrm{Cce}}$ & $0.66 \pm 0.01^{\mathrm{Ba} a}$ & $0.50 \pm 0.01^{\mathrm{Ba} a}$ & $0.23 \pm 0.01^{\mathrm{Bd} d}$ & $1.41 \pm 0.01^{\mathrm{Aa} a}$ & $0.56 \pm 0.01^{\mathrm{Bc} b}$ & $1.23 \pm 0.01^{\mathrm{Ca} b}$ & $0.47 \pm 0.01^{\mathrm{Baa}}$ \\
\hline & 5 & $0.00 \pm 0.0^{\mathrm{B} c c}$ & $0.00 \pm 0.0^{\mathrm{Db} b}$ & $0.75 \pm 0.01^{\mathrm{Bb} c}$ & $2.00 \pm 0.01^{\mathrm{Aa} a}$ & $0.03 \pm 0.01^{\mathrm{Cce}}$ & $0.13 \pm 0.01^{\mathrm{Bb} b}$ & $0.38 \pm 0.01^{\mathrm{Ba} b}$ & $0.36 \pm 0.01^{\mathrm{Bd} b}$ & $0.63 \pm 0.01^{\mathrm{Bb} b}$ & $0.80 \pm 0.01^{\mathrm{Bb} b}$ & $1.19 \pm 0.01^{\mathrm{Ca} b}$ & $0.00 \pm 0.0^{\mathrm{Db} c}$ \\
\hline & 10 & $0.00 \pm 0.0^{\mathrm{Ccc}}$ & $0.00 \pm 0.0^{\mathrm{Cb} b}$ & $0.75 \pm 0.01^{\mathrm{Bb} c}$ & $0.41 \pm 0.01^{\mathrm{Bb} c}$ & $1.13 \pm 0.01^{\mathrm{Aa} a}$ & $0.14 \pm 0.01^{\mathrm{Cb} b}$ & $0.42 \pm 0.01^{\mathrm{Ba} a}$ & $0.42 \pm 0.01^{\mathrm{Bd} b}$ & $0.61 \pm 0.01^{\mathrm{Bb} b}$ & $0.75 \pm 0.01^{\mathrm{Bb} b}$ & $1.13 \pm 0.01^{\mathrm{Ca} b}$ & $0.42 \pm 0.01^{\mathrm{Baa}}$ \\
\hline & 20 & $0.00 \pm 0.0^{\mathrm{D} c c}$ & $0.00 \pm 0.0^{\mathrm{Db} b}$ & $1.95 \pm 0.01^{\mathrm{Aa} b}$ & $0.14 \pm 0.01^{\mathrm{Bb} d}$ & $0.22 \pm 0.01^{\mathrm{Bb} d}$ & $0.06 \pm 0.01^{\mathrm{Ccc}}$ & $0.28 \pm 0.01^{\mathrm{Bb} b}$ & $3.55 \pm 0.01^{\mathrm{Aa} a}$ & $1.09 \pm 0.01^{\text {Аа } a}$ & $1.00 \pm 0.01^{\mathrm{Aa} a}$ & $1.25 \pm 0.01^{\mathrm{Ca} b}$ & $0.23 \pm 0.01^{\mathrm{Ba} a}$ \\
\hline & 30 & $.30 \pm 0.01^{\mathrm{Ca} a}$ & $0.00 \pm 0.0^{\mathrm{Cb} b}$ & $2.06 \pm 0.01^{\mathrm{Aa} a}$ & $0.00 \pm 0.0^{\mathrm{Dc} f}$ & $0.09 \pm 0.01^{\mathrm{Cce}}$ & $0.00 \pm 0.0^{\mathrm{Dd} d}$ & $0.06 \pm 0.01^{\mathrm{Ccc}}$ & $0.73 \pm 0.01^{\mathrm{B} c c}$ & $0.00 \pm 0.0^{\mathrm{Dcd} d}$ & $0.14 \pm 0.01^{\mathrm{Bcc}}$ & $4.38 \pm 0.01^{\mathrm{Aa} a}$ & $0.23 \pm 0.01^{\mathrm{Ba} a}$ \\
\hline & 40 & $0.09 \pm 0.01^{\mathrm{Cb} b}$ & $0.00 \pm 0.0^{\mathrm{Db} b}$ & $1.50 \pm 0.01^{\mathrm{Aa} b}$ & $0.00 \pm 0.0^{\mathrm{Dcf} f}$ & $0.17 \pm 0.01^{\mathrm{Bb} d}$ & $0.09 \pm 0.01^{\mathrm{Ccc}}$ & $0.13 \pm 0.01^{\mathrm{Bb} b}$ & $2.92 \pm 0.01^{\mathrm{Ab} b}$ & $0.00 \pm 0.0^{\mathrm{Dcd} d}$ & $0.06 \pm 0.01^{\mathrm{Cd} d}$ & $0.63 \pm 0.01^{\mathrm{Bce}}$ & $0.22 \pm 0.01^{\mathrm{Ba} a}$ \\
\hline \multirow{6}{*}{2} & 0 & $09 \pm 0.01^{\mathrm{Cb} b}$ & $0.00 \pm 0.0^{\mathrm{Da} b}$ & $1.08 \pm 0.01^{\mathrm{Aa} b}$ & $0.98 \pm 0.01^{\mathrm{Aac}}$ & $0.63 \pm 0.01^{\mathrm{B} a b}$ & $0.09 \pm 0.01^{\mathrm{Cac}}$ & $0.47 \pm 0.01^{\mathrm{Ba} a}$ & $0.42 \pm 0.01^{\mathrm{Bcd}}$ & $0.98 \pm 0.01^{\mathrm{Ab} d}$ & $0.77 \pm 0.01^{\mathrm{Ba} b}$ & $2.81 \pm 0.01^{\mathrm{Ab} b}$ & $0.20 \pm 0.01^{\mathrm{Bab}}$ \\
\hline & 5 & $0.16 \pm 0.01^{\mathrm{Ba} a}$ & $0.00 \pm 0.0^{\mathrm{Ca} b}$ & $1.25 \pm 0.01^{\mathrm{Aa} b}$ & $0.81 \pm 0.01^{\mathrm{Bac}}$ & $0.53 \pm 0.01^{\mathrm{Ba} b}$ & $0.00 \pm 0.0^{\mathrm{Db} d}$ & $0.41 \pm 0.01^{\mathrm{Ba} a}$ & $0.92 \pm 0.01^{\mathrm{Bb} c}$ & $1.00 \pm 0.01^{\mathrm{Aa} a}$ & $0.31 \pm 0.01^{\mathrm{Bb} c}$ & $0.92 \pm 0.01^{\mathrm{Bb} d}$ & $0.25 \pm 0.01^{\mathrm{Ba} a}$ \\
\hline & 10 & $0.13 \pm 0.01^{\mathrm{Ba} a}$ & $0.00 \pm 0.0^{\mathrm{Ca} b}$ & $1.16 \pm 0.01^{\mathrm{Aa} b}$ & $0.38 \pm 0.01^{\mathrm{Bb} d}$ & $0.61 \pm 0.01^{\mathrm{Ba} b}$ & $0.00 \pm 0.0^{\mathrm{Cb} d}$ & $0.28 \pm 0.01^{\mathrm{Bb} b}$ & $0.80 \pm 0.01^{\mathrm{Bb} c}$ & $1.11 \pm 0.01^{\text {Аа } a}$ & $0.89 \pm 0.01^{\mathrm{Ba} b}$ & $0.73 \pm 0.01^{\mathrm{Bc} d}$ & $0.00 \pm 0.0^{\mathrm{Cb} c}$ \\
\hline & 20 & $0.06 \pm 0.01^{\mathrm{Cb} b}$ & $0.00 \pm 0.0^{\mathrm{Ca} b}$ & $0.75 \pm 0.01^{\mathrm{Bb} c}$ & $0.05 \pm 0.01^{\mathrm{Cce}}$ & $0.05 \pm 0.01^{\mathrm{Ce} b}$ & $0.00 \pm 0.0^{\mathrm{Db} d}$ & $0.06 \pm 0.01^{\mathrm{Ccc}}$ & $0.95 \pm 0.01^{\mathrm{Bb} c}$ & $0.36 \pm 0.01^{\mathrm{Bc} b}$ & $0.19 \pm 0.01^{\mathrm{Bb} c}$ & $4.69 \pm 0.01^{\mathrm{Aa} a}$ & $0.00 \pm 0.0^{\mathrm{Db} c}$ \\
\hline & 30 & $0.06 \pm 0.01^{\mathrm{Bb} b}$ & $0.00 \pm 0.0^{\mathrm{Ca} b}$ & $0.94 \pm 0.01^{\mathrm{Ab} c}$ & $0.19 \pm 0.01^{\mathrm{Bb} d}$ & $0.00 \pm 0.0^{\mathrm{Cb} e}$ & $0.00 \pm 0.0^{\mathrm{Cb} d}$ & $0.06 \pm 0.01^{\mathrm{Bcc}}$ & $0.88 \pm 0.01^{\mathrm{Ab} c}$ & $0.06 \pm 0.01^{\mathrm{Bd} c}$ & $0.00 \pm 0.0^{\mathrm{Cd} e}$ & $0.78 \pm 0.01^{\mathrm{Acd}}$ & $0.00 \pm 0.0^{\mathrm{Cb} c}$ \\
\hline & 40 & $0.09 \pm 0.01^{\mathrm{Cb} b}$ & $0.00 \pm 0.0^{\mathrm{Da} a}$ & $0.67 \pm 0.01^{\mathrm{Bb} c}$ & $0.00 \pm 0.0^{\mathrm{Dd} f}$ & $0.00 \pm 0.0^{\mathrm{Cc} f}$ & $0.00 \pm 0.0^{\mathrm{Db} d}$ & $0.00 \pm 0.0 \mathrm{~d}^{\mathrm{Dd} d}$ & $3.61 \pm 0.01^{\mathrm{Aa} a}$ & $0.00 \pm 0.0^{\mathrm{Ded}}$ & $0.09 \pm 0.01^{\mathrm{Ccd}}$ & $3.91 \pm 0.01^{\mathrm{Ab} a}$ & $0.00 \pm 0.0^{\mathrm{Db} c}$ \\
\hline \multirow{6}{*}{3} & 0 & $0.06 \pm 0.01^{\mathrm{Ba} b}$ & $0.00 \pm 0.0^{\mathrm{Da} b}$ & $0.75 \pm 0.01^{\mathrm{Bac}}$ & $0.44 \pm 0.01^{\mathrm{Bb} c}$ & $0.22 \pm 0.01^{\mathrm{Dcf} f}$ & $0.00 \pm 0.0^{\mathrm{Dad} d}$ & $0.14 \pm 0.01^{\mathrm{Bb} b}$ & $0.73 \pm 0.01^{\mathrm{Bb} c}$ & $0.67 \pm 0.01^{\mathrm{Bb} b}$ & $0.33 \pm 0.01^{\mathrm{Bb} c}$ & $5.63 \pm 0.01^{\mathrm{Aa} a}$ & $0.00 \pm 0.0^{\mathrm{Dac}}$ \\
\hline & 5 & $0.03 \pm 0.01^{\mathrm{Ca} b}$ & $0.00 \pm 0.0^{\mathrm{Da} b}$ & $0.75 \pm 0.01^{\mathrm{Bac}}$ & $1.06 \pm 0.01^{\mathrm{Aa} b}$ & $0.42 \pm 0.01^{\mathrm{Bb} d}$ & $0.00 \pm 0.0^{\mathrm{Cad} d}$ & $0.47 \pm 0.01^{\mathrm{Ba} a}$ & $2.00 \pm 0.01^{\mathrm{Aa} b}$ & $1.50 \pm 0.01^{\text {Аа } a}$ & $0.66 \pm 0.01^{\mathrm{Ba} b}$ & $0.73 \pm 0.01^{\mathrm{Bb} d}$ & $0.00 \pm 0.0^{\mathrm{Dac}}$ \\
\hline & 10 & $0.05 \pm 0.01^{\mathrm{Ca} b}$ & $0.00 \pm 0.0^{\mathrm{Da} b}$ & $0.75 \pm 0.01^{\mathrm{Bac}}$ & $0.44 \pm 0.01^{\mathrm{Bb} c}$ & $0.44 \pm 0.01^{\mathrm{Bac}}$ & $0.00 \pm 0.0^{\mathrm{Dad} d}$ & $0.34 \pm 0.01^{\mathrm{Bb} a}$ & $1.14 \pm 0.01^{\mathrm{Aac}}$ & $0.88 \pm 0.01^{\mathrm{Bb} b}$ & $0.61 \pm 0.01^{\mathrm{Ba} b}$ & $0.72 \pm 0.01^{\mathrm{Bb} d}$ & $0.00 \pm 0.0^{\mathrm{Dac}}$ \\
\hline & 20 & $0.00 \pm 0.0^{\mathrm{Bb} c}$ & $0.00 \pm 0.0^{\mathrm{Da} b}$ & $0.56 \pm 0.01^{\mathrm{Bb} c}$ & $0.19 \pm 0.01^{\mathrm{Bc} d}$ & $0.17 \pm 0.01^{\mathrm{Bac}}$ & $0.00 \pm 0.0^{\mathrm{Dad} d}$ & $0.06 \pm 0.01^{\mathrm{Ccc}}$ & $1.13 \pm 0.01^{\mathrm{Aac}}$ & $0.19 \pm 0.01^{\mathrm{Bb} b}$ & $0.06 \pm 0.01^{\mathrm{Ccd}}$ & $0.88 \pm 0.01^{\mathrm{Bb} d}$ & $0.00 \pm 0.0^{\mathrm{Dac}}$ \\
\hline & 30 & $0.00 \pm 0.0^{\mathrm{Cb} c}$ & $0.00 \pm 0.0^{\mathrm{Ca} b}$ & $0.50 \pm 0.01^{\mathrm{Bb} c}$ & $0.19 \pm 0.01^{\mathrm{Bc} d}$ & $0.00 \pm 0.0^{\mathrm{Bb} d}$ & $0.00 \pm 0.0^{\mathrm{Cad} d}$ & $0.00 \pm 0.0^{\mathrm{Cd} c}$ & $3.66 \pm 0.01^{\mathrm{Aa} a}$ & $0.00 \pm 0.0^{\mathrm{Ccd} d}$ & $0.36 \pm 0.01^{\mathrm{Bb} c}$ & $0.48 \pm 0.01^{\mathrm{Bce}}$ & $0.00 \pm 0.0^{\mathrm{Cac}}$ \\
\hline & 40 & $0.00 \pm 0.0^{\mathrm{Cb} c}$ & $0.00 \pm 0.0^{\mathrm{Ca} b}$ & $0.56 \pm 0.01^{\mathrm{Bb} c}$ & $0.00 \pm 0.0^{\mathrm{Cd} f}$ & $0.00 \pm 0.0^{\mathrm{ccf} f}$ & $0.00 \pm 0.0^{\mathrm{Cad}}$ & $0.00 \pm 0.0^{\mathrm{Cd} c}$ & $4.16 \pm 0.01^{\mathrm{Aa} a}$ & $0.20 \pm 0.01^{\mathrm{Bb} d}$ & $0.00 \pm 0.0^{\mathrm{Cd} e}$ & $0.95 \pm 0.01^{\mathrm{Bb} d}$ & $0.00 \pm 0.0^{\mathrm{Cac}}$ \\
\hline
\end{tabular}

** A, B , C , D ..: Capital letters show the difference between the months for each station and the difference between the months carrying different capital letters on the same line is statistically significant $(\mathrm{p}<0.05)$,

$\mathrm{a}, \mathrm{b}, \mathrm{c}, \mathrm{d} . .:$ Lower case letters show the difference between the depths for each station and the difference between the depths carrying different lower case letters in the same line is statistically significant $(\mathrm{p}<0.05)$,

$\mathrm{a}, \mathrm{b}, \mathrm{c}, \mathrm{d} . .$. Italic letters show the difference between stations for each station and the difference between the stations carrying different italic letters in the same line is statistically significant $(\mathrm{p}<0.05)$. 
Table 7. Some morphometric and hydrological parameters of Lake Tortum

\begin{tabular}{c|c|c|c|c}
\hline Parameters & Symbol & Formula & Value & References \\
\hline Drainage area $\left(\mathbf{k m}^{\mathbf{2}}\right)$ & $\mathrm{Ad}$ & - & 1653 & Supplied from DSI \\
Surface area $\left(\mathbf{k m}^{\mathbf{2}}\right)$ & $\mathrm{Ao}$ & - & 6.45 & Supplied from DSI \\
Drainage area/Surface area & - & $\mathrm{Ad} / \mathrm{Ao}$ & 256.28 & Calculated from formula \\
Lake volume $\left(\mathbf{1 0}^{\mathbf{6}} \mathbf{~}^{\mathbf{3}}\right)$ & $\mathrm{V}$ & - & 57.6 & Supplied from DSI \\
Mean depth $(\mathbf{m})$ & $\mathrm{z}$ & - & 100 & Supplied from DSI \\
Total outflow $\left(\mathbf{1 0}^{\mathbf{6}} \mathbf{~ m}^{\mathbf{3}}\right)$ & $\mathrm{Q}$ & & 480 & Supplied from DSI \\
Flushing rate $\left(\mathbf{y r}^{-1}\right)$ & $\mathrm{p}$ & $\mathrm{p}=\mathrm{Q} / \mathrm{V}$ & 8.33 & Calculated from formula \\
Hydraulic retention time $(\mathbf{y e a r})$ & $\mathrm{tw}$ & $\mathrm{tw}=1 / \mathrm{p}$ & 0.12 & Calculated from formula \\
Water load $\left(\mathbf{m ~ y r}^{-1}\right)$ & $\mathrm{q}$ & $\mathrm{q}=\mathrm{Q} / \mathrm{Ao}$ & 74.42 & Calculated from formula \\
Critical phosphorus loading & $\mathrm{Lp}$ & $\mathrm{Lp}=\mathrm{TPL} / \mathrm{Ao}$ & 1.71 & Calculated from formula \\
$\left(\mathbf{g ~ m}^{-\mathbf{2}} \mathbf{y r} \mathbf{r}^{-1}\right)$ & & & \\
\hline
\end{tabular}

\section{Chlorophyll-a}

The changes in the value of chlorophyll-a in different months, stations, and depths were found to be statistically significant $(p<0.05)$. The mean chlorophyll-a value of the lake was $0.04 \mathrm{mgL}^{-1}$, and the lowest value was measured as $0.01 \mathrm{mgL}^{-1}$ in the months of February 2017 and January 2018, whereas the highest value, $0.15 \mathrm{mgL}^{-1}$, was detected in April. When the changes according to the stations and depths are considered, the lowest value at the first station $\left(0.0 \pm 0.0 \mathrm{mgL}^{-1}\right)$ was found at all depths in February and at all depths except the surface in January. The highest value, on the other hand, was $0.31 \pm 0.01 \mathrm{mgL}^{-1}$ in April at $10 \mathrm{~m}$ depth. At the second station, the lowest value $\left(0.0 \pm 0.0 \mathrm{mgL}^{-1}\right)$ was found at all depths in January and February. The highest value, on the other hand, was $0.27 \pm 0.02 \mathrm{mgL}^{-1}$ in April at $5 \mathrm{~m}$ depth. At the third station, the lowest value $\left(0.0 \pm 0.0 \mathrm{mgL}^{-1}\right)$ was observed in November and December, whereas the highest value $\left(0.26 \pm 0.01 \mathrm{mgL}^{-1}\right)$ was in April and at the surface.

Strong correlations were found between chlorophyll-a and $\mathrm{NH}_{3}-\mathrm{N}$ and $\mathrm{PO}_{4}-\mathrm{P}$. On the contrary, the correlation was weak with other parameters. Additionally, there was a negative correlation with TP and $\mathrm{NO}_{3}-\mathrm{N}$ (Table 8).

\section{Phytoplankton composition and biodiversity in Lake Tortum}

In this study, 51 phytoplankton species in total were detected in Lake Tortum; they were Bacillariophyta (38), Charophyta (2), Chlorophyta (7), Cyanobacteria (3) and Pyrrophyta (1). Throughout the investigation period, the most detected species was Ceratium hirundinella. In addition to these, there was a specific increase in species Microcystis aeruginosa and Oscillatoria limosa (Table 9).

Pyrrophyta were detected throughout the study period at the first station. On the other hand, Cyanobacteria species showed an increase only in the autumn. At the second station, all species increased in number in April. A sharp decrease in the number of all species was observed in the summer months. Later, especially in October, the number of individuals from Bacillariophyta, Cyanobacteria, and Pyrrophyta increased. Charophyta had an increase in their number in January. At the third station, Bacillariophyta were observed throughout the study period. Bacillariophyta, Cyanobacteria, and Pyrrophyta were detected increase in the number of species in April, September and October. Additionally, Cyanobacteria were more frequently detected in February and March than were the other division (Fig. 2). 
Table 8. Correlation is TP, $\mathrm{PO}_{4}-\mathrm{P}, \mathrm{NH}_{3}-\mathrm{N}, \mathrm{NO}_{3}-\mathrm{N}, \mathrm{NO}_{2}-\mathrm{N}$ and Chl-a

\begin{tabular}{|c|c|c|c|c|c|c|c|}
\hline & \multicolumn{6}{|c|}{ Correlations } \\
\hline & & TP & $\mathrm{PO}_{4}-\mathrm{P}$ & $\mathrm{NH}_{3}-\mathrm{N}$ & $\mathrm{NO}_{2}-\mathrm{N}$ & $\mathrm{NO}_{3}-\mathrm{N}$ & Chl-a \\
\hline \multirow{3}{*}{$\mathrm{TP}$} & Pearson Correlation & 1 & $.292^{* *}$ & $-.169^{* *}$ & $.202^{* *}$ & $.107^{* *}$ & -.060 \\
\hline & Sig. (2-tailed) & & .000 & .000 & .000 & .002 & .076 \\
\hline & $\mathrm{N}$ & 864 & 864 & 864 & 864 & 864 & 864 \\
\hline \multirow{3}{*}{$\mathrm{PO}_{4}-\mathrm{P}$} & Pearson Correlation & $.292^{* *}$ & 1 & .023 & $-.094^{* *}$ & $-.211^{* *}$ & $.123^{* * *}$ \\
\hline & Sig. (2-tailed) & .000 & & .508 & .006 & .000 & .000 \\
\hline & $\mathrm{N}$ & 864 & 864 & 864 & 864 & 864 & 864 \\
\hline \multirow{3}{*}{$\mathrm{NH}_{3}-\mathrm{N}$} & Pearson Correlation & $-.169^{* * *}$ & .023 & 1 & -.012 & -.030 & $.151^{\text {** }}$ \\
\hline & Sig. (2-tailed) & .000 & .508 & & .735 & .384 & .000 \\
\hline & $\mathrm{N}$ & 864 & 864 & 864 & 864 & 864 & 864 \\
\hline \multirow{3}{*}{$\mathrm{NO}_{2}-\mathrm{N}$} & Pearson Correlation & $.202^{* *}$ & $-.094^{* *}$ & -.012 & 1 & $.570^{* *}$ & .060 \\
\hline & Sig. (2-tailed) & .000 & .006 & .735 & & .000 & .078 \\
\hline & $\mathrm{N}$ & 864 & 864 & 864 & 864 & 864 & 864 \\
\hline \multirow{3}{*}{$\mathrm{NO}_{3}-\mathrm{N}$} & Pearson Correlation & $.107^{* *}$ & $-.211^{* *}$ & -.030 & $.570^{* *}$ & 1 & -.042 \\
\hline & Sig. (2-tailed) & .002 & .000 & .384 & .000 & & .218 \\
\hline & $\mathrm{N}$ & 864 & 864 & 864 & 864 & 864 & 864 \\
\hline \multirow{3}{*}{ Chl-a } & Pearson Correlation & -.060 & $.123^{* *}$ & $.151^{* *}$ & .060 & -.042 & 1 \\
\hline & Sig. (2-tailed) & .076 & .000 & .000 & .078 & .218 & \\
\hline & $\mathrm{N}$ & 864 & 864 & 864 & 864 & 864 & 864 \\
\hline
\end{tabular}

** Correlation is significant at the 0.01 level (2-tailed)

Table 9. Phytoplankton taxa identified in Lake Tortum, 2018

\begin{tabular}{|c|c|}
\hline 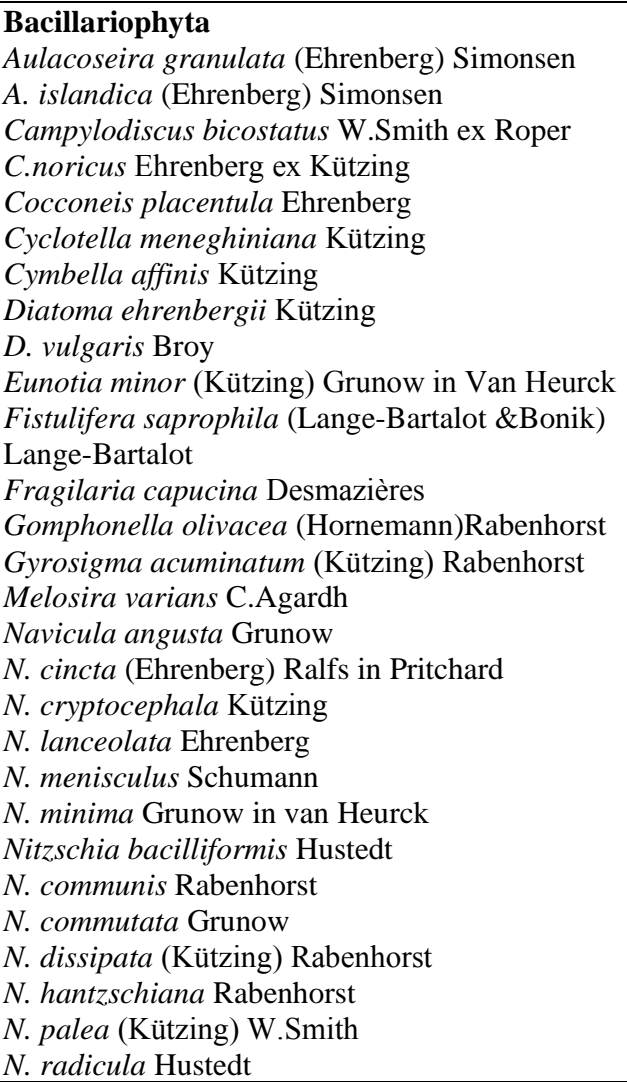 & $\begin{array}{l}\text { N. recta Hantzsch ex Rabenhorst } \\
\text { N. sublinearis Hustedt } \\
\text { Pantocsekiella ocellata(Pantocsek) K.T.Kiss \& Ács in Ács } \\
\text { \& al. } \\
\text { Rhopalodia gibberula (Ehrenberg) Otto Müller } \\
\text { Stephanodiscus neoastraea Hakansson \& Hickel } \\
\text { Surirella brebissonii Krammer \& Lange-Bertalot } \\
\text { S. elegans Ehrenberg } \\
\text { Tryblionella scalaris (Ehrenberg) Siver \& P.B.Hamilton } \\
\text { Ulnaria ulna (Nitzsch) Compère } \\
\text { Chlorophyta } \\
\text { Asterionella formosa } \text { Hassall } \\
\text { Botryococcus braunii Kützing } \\
\text { Chlamydocapsa planctonica (West \& G.S. West) Fott } \\
\text { Chlamydomonas microsphaerella } \text { Pascher \& Jahoda } \\
\text { Chlorotetraedron incus (Teiling) Komárek \& Kovácik } \\
\text { Monoraphidium contortum (Thuret) Komárková-Legnerová } \\
\text { in Fott } \\
\text { Scenedesmus ellipticus } \text { Corda } \\
\text { Charophyta } \\
\text { Staurastrum cingulum } \text { (West \& G.S. West) G.M. Smith } \\
\text { S. diacanthum A.Lemaire } \\
\text { Cyanobakteri } \\
\text { Anabaenopsis circularis (G.S.West) Woloszynska \& } \\
\text { V.V.Miller in V.V.Miller } \\
\text { Microcystis aeruginosa (Kützing) Kützing } \\
\text { Oscillatoria limosa C.Agardh ex Gomont } \\
\text { Pyrrophyta } \\
\text { Ceratium hirundinella (O.F.Müller) Dujardin }\end{array}$ \\
\hline
\end{tabular}




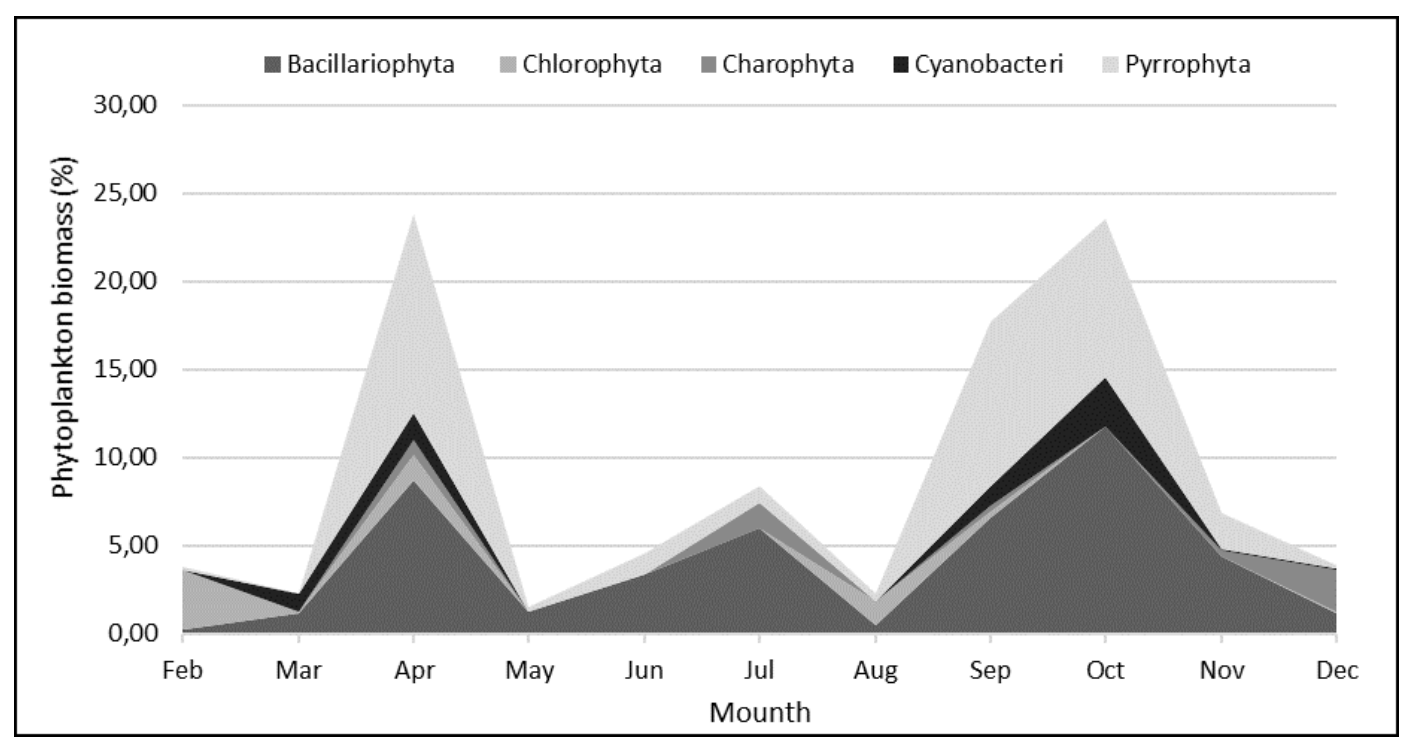

Figure 2. Phytoplankton composition of Lake Tortum

The dominant functional groups were both LM (Ceratium hirundinella) and MP (pennate diatoms) in the Lake Tortum in 2017-2018. Ceratium hirundinella, which is the only species found continuously during the research period, showed an increase in the autumn periods. Cyclotella ocellata (D) showed numerical increases in October and November. The Pennad diatoms, Fragilaria capucina (MP), were observed intensively in September. The other pennad diatoms $N$. saprophila and $N$. palea (MP) were idendificated in spring and early summer. In addition, Microcystis aeruginosa (LM) was found to be increasing in April, May and in October and Oscillatoria limosa (MP) was observed intensively in April (Fig. 3). the Q index indicated tolerable (mean 1.88) ecological conditions. The Q quality index based on stations (1, 2 and 3) were 1.79 (tolerable), 2.21 (medium) and 1.63 (tolerable), respectively (Fig. 4).

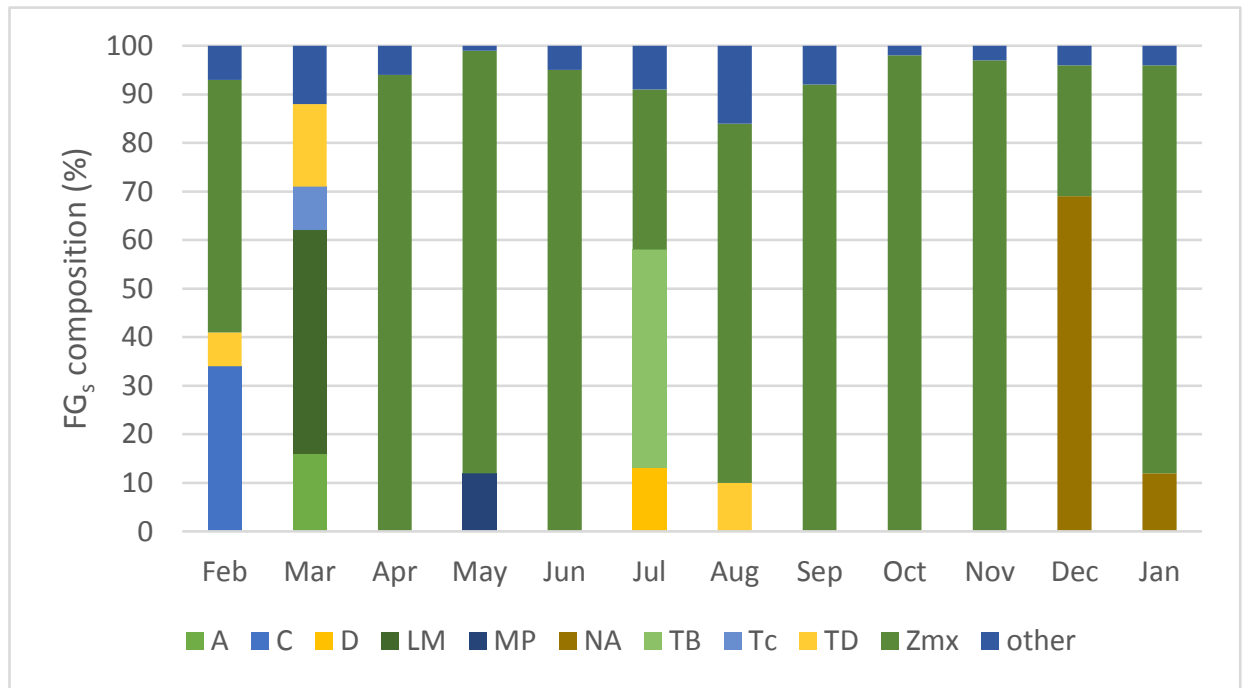

Figure 3. Seasonal succession of phytoplankton functional groups (FGs) in in Lake Tortum. "Others" mean phytoplankton species that constituted less than 5\% of the total biomass 


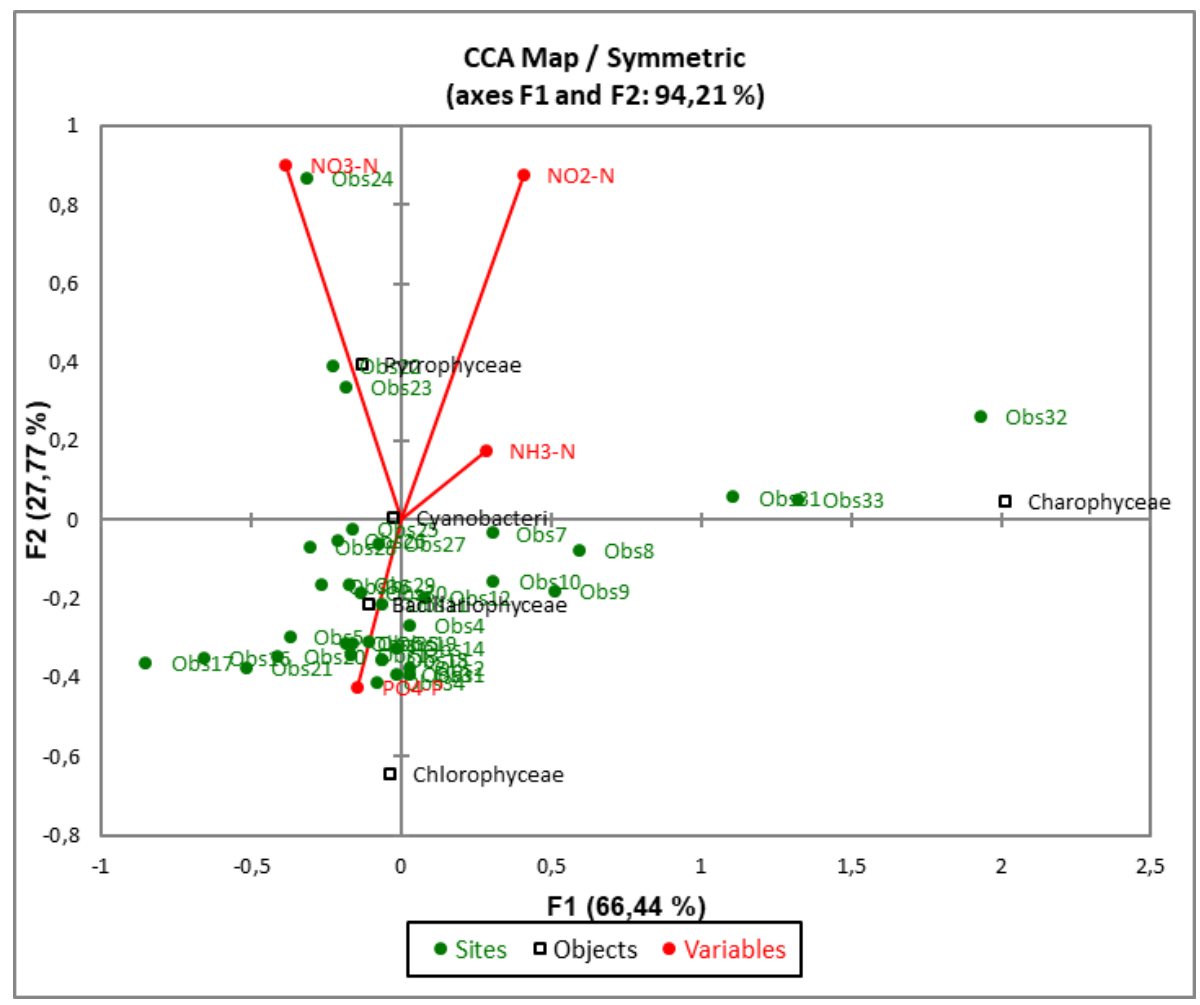

Figure 4. Species-conditional triplot based on a canonical correspondence analysis of the example phytoplankton data displaying $27.77 \%$ of the inertia (= weighted variance) in the abundances and $94.21 \%$ of variance in the weighted averages and class totals of species with respect to the environmental variables

\section{Discussion}

Lake Tortum is a landslide lake; moreover, it is a deep and open lake (Tanyolac, 2009). According to measurements of DSI, the mean depth of lake adopted as $100 \mathrm{~m}$. Previous measurements of mean depth were $110 \mathrm{~m}$ in 1982 (Altuner, 1982), $100 \mathrm{~m}$ in 2003 (Kivrak, 2006), and $80 \mathrm{~m}$ in 2013 (Fakioglu et al., 2014). This lake is the major source for the Tortum Waterfall; for that reason, the regulator in the discharge region of the lake is opened during periods of drought to feed the waterfall. Thus, there may be temporary reductions in the water level. Nevertheless, the studies done in Lake Tortum indicate a $10 \mathrm{~m}$ reduction in depth since 1982.

In this study period, a weak thermal stratification in the lake has been observed. Even though mean water temperature value was measured $6.81 \pm 1.00{ }^{\circ} \mathrm{C}$ in this study, it was measured in 2003 and 2013 as $12.85 \pm 5.75^{\circ} \mathrm{C}$ and $10.5^{\circ} \mathrm{C}$, respectively (Kivrak, 2006; Fakıglu et al., 2014). Warm winters are especially critical to temperate in monomictic lakes, because the temperature affects the duration of the winter and its strength and, consequently, the thermal budget of the lake and the vertical distribution of nutrients and dissolved oxygen (Stratile et al., 2003).

The mean dissolved oxygen value in Tortum Lake was found as $6.81 \pm 1.00 \mathrm{mgL}^{-1}$ during the study. When there was mixing in the lake, an increase in dissolved oxygen concentration at the $40 \mathrm{~m}$ depth was observed. According to Turkish Environmental Legislation for Inland Water Resources Classification, the lake was identificated as II. class waters quality (Anonymous, 2012). The average dissolved oxygen value was found 
$12.85 \pm 5.75 \mathrm{mgL}^{-1}$ in 2006 and $10.5 \mathrm{mgL}^{-1}$ in 2013 (K1vrak, 2006; Fakıglu et al., 2013). Dissolved oxygen value has droped significantly since 2006. Increased organic load in the past years and subsequent organic degradation may have caused a decrease in dissolved oxygen value. However, fishing in the lake has been banned in the past 5 years, which has increased the fish population. In this case, it is thought that it will affect the decrease in oxygen value.

The lakes being in the easily melted area are hard-water lakes and forthis type of lakes the $\mathrm{pH}$ is close to 8.5 (Tanyolaç, 2009). Tortum Lake is located in the basin where landslide events are seen too much and the average $\mathrm{pH}$ value of lake was measured as 8.48. And, according to the total hardness classification of Lawson (1995) for lakes, water of Lake Tortum is in the hard water classification. Plant biomass is particularly high in moderately hard water bodies (Tanyolac, 2009). Therefore, biodiversity in the lake is very low, only two types of chlorophytes were observed in the littoral region, and only 51 phytoplankton species were identified in the lake.

The mean electrical conductivity was $0.289 \pm 0.03 \mathrm{mS} \mathrm{cm}^{-1}$. In another study in Lake Tortum in 2006, the electrical conductivity was found to be $0.308 \mathrm{mS} \mathrm{cm}^{-1}$ (Kivrak, 2006). Natural lakes with discharge points typically have electrical conductivity values between 0.1 and $1 \mathrm{mS} \mathrm{cm}$. (Ozturk, 2014). It is possible to establish a connection between total dissolved solids concentration and electrical conductivity. The more ion and total dissolved solids concentrations in water, the higher electrical conductivity it has (Metcalf and Eddy, 2002). Tortum Stream carries almost 2.5 million $\mathrm{m}^{3}$ of suspended sediment carries to the lake every year (Kopar and Sevindi, 2013).

In deep lakes, changes in depth and vertical stratification alter the water quality (Salmoso et al., 2002). In this study, according to the trophic status range of natural lakes reported by Wetzel (2001) and Anonymous (1982), Tortum Lake, Secchi depth (4.15 m), chlorophyll-a $\left(0.04 \pm 0.0005 \mathrm{mgL}^{-1}\right)$ and total phosphorus concentration $\left(0.31 \pm 0.03 \mathrm{mgL}^{-1}\right)$, was calculated as mesotrophic. In 1982, the lake was reported to be in an oligotrophic state (Altuner, 1982), and it was pointed to be oligo-mesotrophic in 2003, as well (Kivrak, 2006). Unfortunately, the studies on this lake are very limited. In addition, not all physico-chemical parameters of the lake have been studied in these previous studies. However, these studies cover only certain periods, not every period. Although it makes it difficult for us to understand the change in the lake very clearly, collecting the data of the lake is important for the future of the lake.

In terms of TP values, the lake's trophic level changed from the oligotrophic to the mesotrophic level. In deep lakes, such trophic level changes are under the control of both climatologic and anthropologic variables (Salmoso et al., 2002). The highest TP value was seen on the surface at the first station. At the second and third stations, especially during the time when the mixing is strong (such as in summer months), the $40 \mathrm{~m}$ depth showed high TP values. Phosphorus has a tendency to precipitate on sediments in the particle state (Pulatsu and Topcu, 2012). Besides, the TP also depends on the geologic structure of the region and on organic matter entry into the water (Wetzel, 2001; Tanyolac, 2009). The mean orthophosphate concentration was found to be $0.1 \pm 0.01 \mathrm{mgL}^{-1}$ in this study. That value was $0.05 \mathrm{mg} / \mathrm{L}$ in a study done in 2003 in Lake Tortum (Kivrak, 2006). According to Wetzel (2001), increased zooplankton numbers come from increased orthophosphate concentration, whereas Ozkundakçı (2014) claimed that they were affected by agricultural pollution. Zooplankton species and number were observed quite abundant over the study period. While a fraction of the phosphorus is expected to precipitate on sediments in deep and monomictic Lake Tortum, another part 
again returns to the water column according to the sedimentation, disintegration, and transformation ratios. Moreover, the concentrations of $\mathrm{Fe}, \mathrm{Al}, \mathrm{Cd}$, and $\mathrm{Pb}$ elements were found to be low in heavy metal analyses conducted in Lake Tortum water, yet the potential ecological risk coming from those elements together has been determined to be moderate (Kaya et al., 2017). The increase in total phosphorus value in the lake, led to an increase in the number and variety of the primary producers of the lake. At the first station, where the lake is fed, the chlorophyll $a$ value in summer months at the $40 \mathrm{~m}$ depth was found to be $0.03 \pm 0.01 \mathrm{mgL}^{-1}$, while at other stations in the spring months and in the epilimnion stratum (the upper thermal layer of the lake), it was $0.13 \pm 0.02 \mathrm{mgL}^{-1}$. Similar situations were also observed in other deep lakes, Lakes Gordo and Iseo; in both of them, when the temperature rose in the summer months, both the algae biomass and the level of nutrient salts increased (Salmaso, 2002).

In this study, the $\mathrm{NH}_{3}-\mathrm{N}$ mean value was found to be $0.14 \pm 0.07 \mathrm{mgL}^{-1}$. Fakioglu et al. (2014) and Kivrak (2006) were found that value to be $0.15 \mathrm{mgL}^{-1}$ and $0.19 \pm 0.01 \mathrm{mgL}^{-1}$ in 2013 and 2006, respectively. The mean $\mathrm{NO}_{2}-\mathrm{N}$ concentration and $\mathrm{NO}_{3}-\mathrm{N}$ concentration were calculated $0.55 \pm 0.08 \mathrm{mgL}^{-1}, 1.10 \pm 0.02 \mathrm{mgL}^{-1}$, respectively. An increase was observed for those values in comparison to $\mathrm{NO}_{2}-\mathrm{N}\left(0.07 \mathrm{mgL}^{-1}\right.$, $\left.0.002 \pm 0.0 \mathrm{mgL}^{-1}\right)$ and $\mathrm{NO}_{3}-\mathrm{N}\left(0.48 \mathrm{mgL}^{-1}, 0.08 \pm 0.01 \mathrm{mgL}^{-1}\right)$ values found in 2013 and 2003. In this case, we can say that the amount of ammonia-nitrogen coming to the lake is decreased or the ammonia-nitrogen might be converted to nitrite-nitrogen by the nitrobacterias. Approximately $92 \%$ of the villages around the lake were built on the mountain slopes. The lands that have flatness are used as agricultural lands which contain rich alluvium material (Kopar and Sevindi, 2013). In addition, the increase in nitrite concentration is important as it is an indicator of pollution caused by organic or industrial waste (Pulatsu et al., 2014).

Reduction of the external phosphorus load to the lake is a very crucial step in eutrophication control. There are several studies illustrating the success of that process (Cole, 1983; Oenema, 1991). In Lake Tortum, phosphorus loading was calculated to be $11050 \mathrm{~kg} \mathrm{yr}^{-1}$ (Equation 5). That value is much higher than the critical phosphorus loading value calculated by the formula of Vollenweider (1976). Two main factors on the external phosphorus loading are agricultural activities and the absence of sewer systems (Bronmark and Hansson, 2017). There have been intense reclamation and road expansion activities in recent years on Tortum Stream. Besides, sewer systems are absent in many regions in the vicinity of Tortum Stream and its tributaries. This study found the main component of the TP to be the artificial artifical phosphorus loading. The domestic load was calculated $9707 \mathrm{~kg} \mathrm{yr}^{-1}$ (Equation 4). In the studies carried out in Mogan Lake, domestic phosphorus load was found $2998 \mathrm{~kg} \mathrm{yr}^{-1}$ in 2002 (Pulatsü and Aydın, 1997). In another study conducted on the same lake, domestic phosphorus load was determinated $8084 \mathrm{~kg} \mathrm{yr}^{-1}$ in 2004 (Fakıoglu and Pulatsu, 2004). Domestic phosphorus load value of the study was found to be higher than those values in Lake Mogan, which is a eutrophic lake status. While Lake Mogan is not only a shallow lake, but also under effect of agriculture and anthropogenic pollution, Tortum Lake is both deep and many of these effects are not seen on the shore of the lake. Nevertheless, the external phosphorus load calculated for Tortum Lake was found to be high. This is thought to be a threat to the lake. On the other hand, for the potential ecological risk evaluation of the lake, it was found to be under moderate risk threat; however, while the contamination load risk should be 0 , it was found between 0 and 1 (Kaya et al., 2017). 
The phosphorus export coefficient was calculated to be $1.71 \mathrm{~g} \mathrm{~m}^{-2} \mathrm{yr}^{-1}$ (Equation 6) for Lake Tortum in this study. Even though that value is less than the critical load of $2.17 \mathrm{~g} \mathrm{~m}^{-2} \mathrm{yr}^{-1}$ (Equation 9), it is still higher than the permissible phosphorus export coefficient (0.96 $\mathrm{g} \mathrm{m}^{-2} \mathrm{yr}^{-1}$, Equation 8). Kirchner and Dillon (1975) considered a field with a phosphorus export coefficient between 1.41 and $14.88 \mathrm{~g} \mathrm{~m}^{-2} \mathrm{yr}^{-1}$ to be a forest field and pasture. Lake Tortum is in that category. However, the lake was determinated as oligotrophic status according to the Dillon and Rigler (1975) classification. The phosphorus export coefficient was found to be lower than the critical value of Wetzel (2001) for agricultural and forestry fields.

The reaction of deep lakes to the reduction of external nutrient load has been reported to be faster than that of shallow lakes (Beklioğlu, 1999). Harper (1992) stated that permissible phosphorus loading is $0.4 \mathrm{~g} \mathrm{~m}^{-2} \mathrm{yr}^{-1}$ and that hazardous phosphorus loading is $0.8 \mathrm{~g} \mathrm{~m}^{-2} \mathrm{yr}^{-1}$ for $100 \mathrm{~m}$ deep lakes. Thus, Lake Tortum's phosphorus load is higher than the hazardous level. Even though the nutrient status of the lake was determined to be mesotrophic in terms of TP concentration, unless the necessary precautions are taken the lake might rapidly transform into a eutrophic state. In another deep lake, Lake Bolsena, it was stated that remediation of the lake will take a very long time due to the increase in the external phosphorus loading and the fact that the increase also raised algae production and mineralization in the hypolimnion, the lower thermal layer of the lake (Mosello et al., 2018).

Phytoplankton communities react to the changes in the lake environment rapidly, while they are also in a rivalry for seasonal succession, which is why alterations in the environmental conditions lead to huge compositional diversity (Scheffer et al., 2003). In this study, 51 phytoplankton were detected. Altuner (1982) investigated the phtoplankton in the lake in 1981, and 35 species in total were detected from Bacillariophyta, Chlorophyta, Cyanophyta, Dinophyta, and Crysophyta. Another researcher examined the phytoplankton community in the lake in 2006 and found only 12 species, mainly from Bacillariophyta (Kıvrak, 2006). Lake Salda and Lake Burdur are a deep and highly alkaline lakes of Turkey. These lakes were found at the oligotrophic level due to low nutrient and chlorophyll-a measurements. In both lakes, the taxa of phytoplankton were low (15 taxa in Lake Salda, 21 taxa in Lake Burdur) (Kazanci et al., 2004; Girgin et al., 2004).

The dominant phytoplankton species change according to the nutrient level of the lake. In Lake Tortum, species Microcystis aeruginosa shows an increase in number from time to time. Desmidiaceae and centric diatoms indicate that the lake is oligotrophic, and pennate diatoms and cyanobacteria, on the other hand, are an indicator of a eutrophic lake (Harper, 1992). Species from both groups have been observed in Lake Tortum. The phytoplankton and benthic algae compositions were detected in 1979-1981 as oligotrophic. The researchers observed a high number of Cyclotella kützingenia, a centric diatom, and, albeit in low numbers, Ceratium hirundinella and Microcystis spp. (Altuner, 1982). On the other hand, in a study done in 2002-2003, the dominant species were determined to be Chlamydomonas microsphaerella, Cyclotella krammeri, C. glomerate, and Ceratium hirundinella, while the rare species were Stephanodiscus rotula, Fragilaria ulna, Cocconeis placentula, Cymbella affinis, Navicula salinarum, Carteria spp., Staurastrum vestitum, Trachelomonas volvocina, and Peridinium cinctum (K1vrak, 2006).

The most detected species in our study was Ceratium hirundinella. The second and third were Cyclotella meneghiniana and Fragilaria capucina. According to Reynolds et 
al. (2002) Cyclotella spp. generally found in oligotrophic lakes, but Padisák et al. (2009) reported that this species has mesotrophic or eutrophic lakes. Although Ceratium hirundinella was reported as a species found in nutrient-rich oligotrophic and mesotrophic lakes in summer months (Reynolds et al., 2002), the highest number they reached in this study was seen in October. Some researchs were turn $C$. hirundinella considered as oligotrophic and mesotrophic lakes; while others were evaluated as eutrophic to hypertrophic, small to medium-sized lakes (Padisák et al., 2009; Aboim et al., 2020). However, Dinoflagellata taxa often have been found in eutrophic and mesotrophic lakes of Turkey (Gönülol and Obal1, 1986; Taş et al., 2002; Yerli et al., 2012). Moreover, the indicators of nutrient-rich turbid lakes, Ulnaria ulna and Nitzschia spp. (Padisák et al., 2006), were also detected in this study. Besides, Anabaena elenkinii (code: H1), Microcystis aeruginosa (code: LM), and Oscillatoria limosa (code: MP), belonging to Cyanobacteria, and Chlamydomonas microsphaerella and Asterionella formosa (code: C), belonging to Chlorophyta, were also observed. They are indicators of eutrophic water bodies (K1vrak, 2006). Reynolds et al. (2002) indicate that Asterionella formosa, Aulacoseira granulata (code: P), and cyanobacteria are typical indicators of eutrophic lakes. In Lake Tortum, Asterionella formosa, Aulacoseira granulata, and Microcystis aeruginosa species were detected. In 2014, a yellow area was spotted in the lake, and, when it was investigated, a rapid increase in the numbers of Asterionella formosa was found.

When functional groups were examined on a month-by-month scale, dominant functional groups were found to be LM (C. hirundinella, Microcystis) in April, October and November, MP (Diatome, Navicula, Cymbella, Oscillatoria) in March, C (Asterionella formosa) in Februvary, B (Cyclotella meneghiniana) in March and TB (Fragilaria, Nitzschia) in July. In Lake Mogan, the dominant phytoplankton functional groups of the study period were X2, H1, Y, LM, F, Lo, M, W1, C, P, X1, and S1 and the Q index was indicated a moderate ecological status for Lake Mogan (Demir et al., 2014). Lake Tortum was determined to be mesotrophic according to chemical parameter and chlorophyll-a. Hovewer, Q index was indicated tolerable ecological quality status. The reason for this is that $C$. hirundinella, which is located in Lake Tortum, is evaluated as a functional group in not only oligotrophic but also eutrophic lakes.

Q index has changed in bad ecology except in February and August (clean water phase) in $1^{\text {th }}$ station due to seasonal change in stations. Similarly, it was observed in $3^{\text {rd }}$ station, but this station was in excellent ecological condition in July. It is thought to be the reason that the high phosphorus loading to the lake affects the biodiversity in the $1^{\text {st }}$ station, while filling the lake in $3^{\text {rd }}$ station and the use of these areas as recreational areas. The $2^{\text {nd }}$ station is in the moderate ecological state. Since Tortum Lake is in the deep lake class, it is thought that the ecological status of this station is better than the others. Water quality variables and phytoplankton were examined within the Jequitinhonha River lower course. Results indicated that chemical oxygen demand, dissolved aluminum, and turbidity were the main factors which influenced phytoplankton community structure and composition (Aboim et al., 2020).

In this study, the highest number of total phytoplankters by count was found in April whereas the lowest count was in January. In Lake Tortum, the TP load was found to be higher than the critical phosphorus load. In addition to that, $\mathrm{NO}_{2}-\mathrm{N}$ and $\mathrm{NO}_{3}-\mathrm{N}$ were also high in concentration. As a result, both the species biodiversity and their numbers increased. In the development of Charophyta was effected $\mathrm{NO}_{3}-\mathrm{N}$ value and in Pyrrophyta were found to play a role $\mathrm{NO}_{3}-\mathrm{N}$ and $\mathrm{NH}_{3}-\mathrm{N}$ values. TP and $\mathrm{PO}_{4}-\mathrm{P}$ were found to be a 
limiting factor in species in Bacillariophyta and Chlorophyta. Cyanobacteria group algae were calculated as limiting both nitrogen and phosphorus fractions (Fig. 5). However, Pedators are known to be the main factor in the phytoplankton species diversity and numerical increase as well as nutrient salts. After the rapid increase in the number of phytoplankton in April, there was a sharp decline in May, which can be attributed to the grazing pressure coming from zooplankton at that time, which was also seen in October. It has been reported that, after the increase in phytoplankton and subsequent decrease in nutrients and the filtration of the zooplankton, a clear-water state can arise (Lampert and Sommer, 1997). On the other hand, after the increase in the TP load and, consequently, the increase in phytoplankton and suspended algae (and production) in the lake, there may be a shift in phytoplankton species to the toxic ones or the ones that have not been grazed efficiently by hunter species. That may result in an algal bloom. In addition to that, the increase in phosphorus loading causes an increase in production, biomass, and microalgae composition (Dogan-Saglamtimur and Saglamtimur, 2018).

\section{Conclusion}

It is important to protect and to continuously monitor inadequate freshwater resources and to control the changes that can occur in the lakes as well. Deep and meromictic lakes are especially problematic in regard to restoring their trophic states. It is reported that, in temperate climates where meromictic lakes are prevalent, global warming has a stronger and more steady impact on stratification and, consequently, on lake restoration (Lepori et al., 2018). The works done on Lake Tortum, such as island creation by adding earth and degradation of the shoreline, along with the destructions on Tortum Stream, have significantly damaged the lake ecosystem. Lake Tortum's contamination increases every day. For that reason, species composition in the lake has transformed with time, and more and more eutrophic species have started to appear and become dominant species. In conclusion, the detection of the mechanisms affecting the ecology, hydrology, and morphology of deep lakes is critical for protecting these aquatic systems.

In the future studies are recommend that comprehensive determination of carbon, nitrogen and phosphorous substance loads entering the lake from external point and spread sources, and the use of lake models (mathematical model), including bioprocesses (carbonaceous removal, nitrification, denitrification, photosynthesis) and other physical (sedimentation, resuspension) and chemical processes (adsorption, chemical precipitation) that have an impact on the consumption and production of the lake.

Acknowledgements. This work was supported by the Scientific and Technological Research Council of Turkey (TUBITAK) (grant number 116Y261, 2018).

\section{REFERENCES}

[1] Aboim, I. G., Gomes, D. F., Junior, P. O. M. (2020): Phytoplankton response to water quality seasonality in a Brazilian neotropical river. - Environ Monit Assess 192(70): 3-16.

[2] Altuner, Z. (1982): A Research on Phytoplankton and Benthic Algae in Lake Tortum. Atatürk University, Institute of Science and Technology, Erzurum, PhD Thesis, 83p. (in Turkish).

[3] Anonymous (1982): Eutrophication of Waters. - Monitoring, Assessment and Control, OECD, 154p., Paris. 
[4] Anonymous (1995): Standard Methods for the Examination of Water and Wastewater. 19th ed., American Public Health Association (APHA), 1193p., Washington.

[5] Anonymous (2003): Water Quality-Guidance Standard for the Routine Analysis of Phytoplankton Abundance and Composition Using İnverted Microscopy (Utermöhl Technique). - CEN TC 230/WG 2/TG 3/N73, 37p.

[6] Anonymous (2012): Water Quality Criteria for Turkey by class of inland water resources according to environmental legislation. - Surface Water Quality Management Regulation, First Part, Ministry of Forestry and Water Management, Turkey.

[7] Bronmark, C., Hansson, L. A. (2017): The biology of lakes and ponds. - Oxford University Press, 368p.

[8] Chapra, S. C., Tarapchack, S. J. (1976): A clorophyll a model and its relation to phosphorus loading plots for lakes. - Water Resour. Res. 12(6): 1260-1264.

[9] Coban, F. (2007): Investigation of the Water Quality of the Hazar Lake. - Master Thesis, Environmental Engineering, Elazığ, Turkey. (in Turkish).

[10] Cole, G. A. (1983): Textbook of Limnology. - The C. U. Mosloy Company, 404p., U.S.A.

[11] Cox, E. J. (1996): Identification of Freshwater Diatoms from Live Material. - Chapman and Hall, 158p. London.

[12] Demir, A. N., Fakioglu, O., Dural, B. (2014): Phytoplankton functional groups provide a quality assessment method by the Q assemblage index in Lake Mogan (Turkey). - Turkish Journal of Botany 38(1): 169-179.

[13] Dillon, P. J., Rigler, F. H. (1975): A Simple Metod for Predicting the Capacity of a Lake for Development Based On Lake Trophic Status. - Fish. Res. Board Can. 32(9): 15191531.

[14] Dogan-Saglamtimur, N., Saglamtimur, B. (2018): Eutrophication Status and Scenarios in Aquatic Environments. - Ömer Halisdemir Üniversitesi Journal of Engineering Sciences 7(1): 75-82. (in Turkish).

[15] Duman, T. Y. (2009): The Largest Landslide Dam in Turkey: Tortum landslide. Engineering Geology 104(1-2): 66-79.

[16] Fakioglu, O., Pulatsu, S. (2004): Determination of External Phosphorus Loading in Mogan Lake (Ankara) Following Some Restoration Measures. - Yuzuncu Y1l University, Faculty of Agriculture, Journal of Agricultural Sciences (J. Agric. Sci.) 15: 63-69. (in Turkish).

[17] Fakığlu, O., Köktürk, M., Atamanalp, M. (2013): The Application of some Biodiversity Indices in the Tortum Stream, Erzurum, Turkey. - International Journal of Physical Science 46: 2069-2076.

[18] Fakıoglu, O., Demir, S. M., Kokturk, M. (2014): Vertical Change of Same PhysicoChemical Parameters in Tortum Lake (Erzurum/Turkey). - Jornal of Animal and Veterinary Advances 13: 864-870.

[19] Fakıglu, O., Köktürk, M., Uzundumlu, A. S., Arslan, H., Atamanalp, M. (2018): Planktonic-based assessment of the landside-dammed lake (Erzurum, Turkey). - Iranian Journal of Fisheries Sciences 17(3): 503-515.

[20] Girgin, S., Kazanc1, N., Dügel, M. (2004): On the limnology of deep and saline Lake Burdur in Turkey. - Acta hydrochimica et hydrobiologica 32(3): 189-200.

[21] Gönülol, A., Obalı, O. (1986): Phytoplanton of the Karamık Lake, Afyon, Turkey. Commun, Fac. Sci. Univ. Ank., Ser. C-4: 105-128.

[22] Harper, D. M. (1992): Eutrophication of freshwaters. - Chapman \& Hall, London, print ISBN 978-94-010-5366-2, 327p.

[23] Huber-Pestalozzi, G. (1938): Das Phytoplankton des Süsswassers, 1 Teil. Blaualgen, Bakterien, Pilze. - In: Thienemann, A. (ed.) Die Binnengewasser. E. Schweizerbart'sche Verlagsbuchhandlung, 342p., Stuttgart. (in German).

[24] Huber-Pestalozzi, G. (1942): Das Phytoplankton des Süsswassers, 2 Teil. Diatomeen. - In: Thienemann, A. (ed.) Die Binnengewasser. E. Schweizerbart'sche Verlagsbuchhandlung, 549p., Stuttgart. (in German). 
[25] Huber-Pestalozzi, G. (1950): Das Phytoplankton des Süsswassers, 3 Teil. Cryoptophyceen, Chloromonadien, Peridineen. - In: Thienemann, A. (ed.) Die Binnengewasser. E. Schweizerbart'sche Verlagsbuchhandlung, 310p., Stuttgart. (in German).

[26] Hustedt, F. (1930): Bacillariophyta (Diatomeae). Heft 10. - In: Pascher, A. (ed.) Die Süsswasser-Flora Mitteleuropas. Verlag von Gustav Fisher, Jena, 466p. (in German).

[27] Jeppesen, E., Kronvang, B., Meerhoff, M., Sondergaard, M., Hansen, K. M., Andersen, H. E., Lauridsen, T. L., Liboriussena, L., Beklioglu, M., Özen, A., Olesen, J. E. (2009): Climate Change Effects on Runoff, Catchment Phosphorus Loading and Lake Ecological State, and Potential Adaptations. - Journal of Environmental Quality 38(5): 1930-1941.

[28] John, D. M., Whitton, B. A., Brook, A. J. (2002): The Freshwater Algal Flora of The British Isles. - Cambridge Univ. Press, Cambridge, 702p.

[29] Kaya, H., Erginal, G., Cakır, Ç., Gazioglu, C., Erginal, A. E. (2017): Ecological risk evaluation of sediment core samples, Lake Tortum (Erzurum, NE Turkey) using environmental indices. - International Journal of Environment and Geoinformatics 4(3): 227-239.

[30] Kazanci, N., Girgin, S., Dügel, M. (2004): On the limnology of Salda Lake, a large and deep soda lake in southwestern Turkey: future management proposals. - Aquatic Conservation: Marine and Freshwater Ecosystems 14(2): 151-162.

[31] Kivrak, E. (2006): Seasonal and Long Term Changes of the Phytoplankton in The Lake Tortum in Relation to Environmental Factors, Erzurum, Turkey. - Biologia 4: 339-345.

[32] Kivrak, E., Gürbüz, H. (2010): The Relationship Between Epipelic Diatoms and Some Physicochemical Properties of Tortum River (Erzurum). - Ekoloji 19(74): 102-109. (in Turkish).

[33] Komarek, J., Anagnostidis, K. (1999): Cyanoprokaryota 1. Teil: Chroococcales. - In: Ettl, H., Gartner, G., Heynig, H., Mollenhauer, D. (eds.) Süsswasserflora von Mitteleuropa. Spektrum Akademischer Verlag, 548p., Heidelberg. (in German).

[34] Kopar, I., Sevindi, C. (2013): Zone-shoreline changes due to current sedimentation and siltation in southwest of Tortum Lake (Uzundere-Erzurum). - Turkish Journal of Geography 60: 49-66. (in Turkish).

[35] Krichner, W. B., Dillon, P. J. (1975): An emprical metod of estimating retention of phosphorus in lakes. - Wat. Resour. Res. 11(1): 82-183.

[36] Lampert, W., Sommer, U. (1997): Limnoloecology: The Ecology of Lakes and Streams. Oxford University Press 382p.

[37] Lawson, T. B. (1995): Water Quality and Environmental Requirements. - In: Fundamentals of Aquacultural Engineering, Springer, Boston, MA.

[38] Lepistö, L., Rosenström, U. (1998): The Most Typical Phytoplankton Taxa in Four Lakes in Finland. - Hydrobiologia 369/370: 89-97.

[39] Lepori, F., Bartosiewicz, M., Simona, M., Veronesi, M. (2018): Effects of winter weather and mixing regime on the restoration of a deep perialpine lake (Lake Lugano, Switzerland and Italy). - Hydrobiologia 824: 229-242.

[40] Lind, M. E., Brook, A. J. (1980): A key to the Commoner Desmids of the English Lake District. Freswater Biol. - Assoc. Publ., 123, Cumbria.

[41] Metcalf, E., Eddy, M. (2002): Wastewater Engineering: Treatment and Resource Recovery. - 4th edition, Mic Graw-Hill, USA. 1816p.

[42] Mosello, R., Bruni, P., Rogora, M., Tartari, G., Dresti, C. (2018): Long-term change in the trophic status and mixing regime of a deep volcanic lake (Lake Bolsena, Central Italy). Limnologica 72: 1-9.

[43] Nygaard, G. (1949): Hidrobiological studies on some Danish ponds and lakes. Part II: The quotient hypothesis and some little known plankton organisms. - Kongelige Danske Videnskabernes Selskab Biologiske Skrifter 7: 1-293.

[44] Oenema, O. (1991): Nitrogen and phosphorus Budgets Indutch Agriculture and Farm Strategies to Increasefertilizer Efficiency. - Wat. Sci. Tech. 24(10): 341-342. 
[45] Ozden, B. G. (2002): Determination of Water Quality of Lake Köyceğiz and Investigation of Eutrophic Level According to Vollenweider Model. - Hacettepe University Faculty of Science Publications, pp.1-132., Ankara, Turkey. (in Turkish).

[46] Ozkundakci, D., Hamilton, D. P., Kelly, D., Schallenberg, M., de Winton, M., Verburg, P., Trolle, D. (2014): Ecological integrity of deep lakes in New Zealand across anthropogenic pressure gradients. - Ecological Indicators 37: 45-57.

[47] Ozturk, B. Y. (2014): Research on the Agora of Apa Dam Lake (Çumra / Konya). - Selcuk University, Institute of Science and Technology. PhD Thesis, 236p. (in Turkish).

[48] Padisák, J., Borics, G., Grigorszky, I., Soróczki-Pintér, É. (2006): Use of Phytoplakton Assemblages for Monitoring Ecological Status of Lakes Within the Water Framework Directives: the assemblage index. - Hydrobiologia 553: 1-14.

[49] Padisák, J., Crossetti, L. O., Naselli-Flores, L. (2009): Use and misuse in the application of the phytoplankton functional classification: a critical review with updates. - Hydrobiologia 621(1): 1-19.

[50] Popovski, J., Pfiester, L. A. (1990): Dinophyceae (Dinoflagellida), Band 6. - In: Ettl, H., Gerloff, J., Heynig, H., Mollenhauer, D. (eds.) Süsswasserflora von Mitteleuropa. Gustav Fishre Verlag, 243p., Jena. (in German).

[51] Prescott, G. W. (1973): Algae of the Western Great Lakes Area. - 5th ed., WM. C. Brown Co. Publ, 977p., Dubuque.

[52] Pulatsu, S., Aydın, F. (1997): Water Quality and Phosphorus Budget of Mogan Lake, Turkey. - Acta Hydrochim. Hydrobiol. 25(3): 128-134. Doi:10.1002/aheh.19970250303.

[53] Pulatsu, S., Topcu, A. (2012): Water Quality in Fish Production. - Ankara University Fisheries Engineering Department. Textbook No: 1591. Ankara University Press, 90p. (in Turkish).

[54] Pulatsu, S., Topcu, A., Atay, D. (2014): Water Pollution and Control. - Ankara University Fisheries Engineering Department Ankara. Textbook No: 1617. Ankara University Press, 384p. (in Turkish).

[55] Reynolds, C. S., Huszar, V., Kruk, K., Naselli-Flores, L., Melo, S. (2002): Towards Classification of the Freshwater Phytoplankton. - Journal of Plankton Research 24: 417428.

[56] Round, F. D. (1953): An Investigation of two Benthic Algal Communities in Malham Tarn. - Yorkshire. J. Ecol. 41: 174-197.

[57] Salmaso, N. (2002): Ecological Patterns of Phytoplankton Assemblages in Lake Garda: Seasonal, Spatial and Historical Features. - Journal of Limnology 61(1): 95-115.

[58] Scheffer, M., Reinaldi, S., Huisman, J., Weissing, F. J. (2003): Why plankton communities have no equilibrium: solutions to the paradox. - Hydrobiologia 491: 9-18.

[59] Selek, Z., Karaaslan, Y. (2019): Ecosystem-Based Water Quality Management. - Ministry of Agriculture and Forestry, e-ISBN No: 978-605-82367-0-7, 490p., Ankara, Turkey. (in Turkish).

[60] Starmach, K. (1966): Cyanophyta. - Flora Slodkowodna Polski, 807p., Warszawa.

[61] Straile, D., Jöhnk, K., Henno, R. (2003): Complex effects of winter warming on the physicochemical characteristics of a deep lake. - Limnology and Oceanography 48(4): 1432-1438.

[62] Strickland, J. D. H., Parssons, T. R. (1972): A Practical Handbook of Seawater Analysis. $2^{\text {nd }}$ ed., Bull. Fish. Res. Board. Can., 311p., Canada.

[63] Tanyolac, J. (2009): Limnology. - Hatipoğlu Press, 294p. Anakara, Turkey. (in Turkish).

[64] Tas, B. I., Gonulol, A., Tas, E. (2002): A study on the seasonal variation of the phytoplankton of Lake Çernek (Samsun-Turkey). - Turkish Journal of Fisheries and Aquatic Sciences 2(2): 121-128.

[65] Thunmark, S. (1945): Zur Soziologie des Süsswasserplanktons. Eine methodisch ökologische Studie. - Folia Limnologica Skandinavica 3: 1-66. 
[66] Utermohl, H. (1958): Zur Vervolkommnung deer quantitativen Phytoplankton-Methodik. Mitteilungen der Internationale Vereinigung der theoretretische und Angewandte. Limnologie 5: 567-596. (in German).

[67] Van Damme, S., Meire, P., Gommers, A., Verbeeck, L., Van Cleemput, E., Derous, S., Degraer, S., Vincx, M. (2007): Typology, Reference Condition and Classification of the Belgian Coastal Waters. - EV/40,SPSDII. D/2007/1191/12. 119p.

[68] Vollenweider, R. A. (1976): Advences in Defining Critical Loading Levels for Phosphorus in Lake Eutrophication. - Mem. Ist. Ital. Hidrobiol. 33: 53-83.

[69] Wetzel, R. G. (2001): Limnology. - W.B. Sounders Company, London, 743p.

[70] Yerli, S. V., Kıvrak, E., Gürbüz, H., Manav, E., Mangit, F., Türkecan, O. (2012): Phytoplankton community, nutrients and chlorophyll-a in Lake Mogan (Turkey); with comparison between current and old data. - Turkish Journal of Fisheries and Aquatic Sciences 12(1): 95-104. 